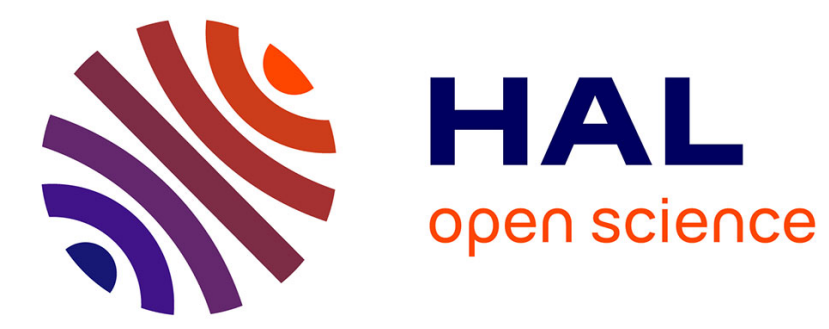

\title{
Hidden bifurcation in the Multispiral Chua Attractor
}

\author{
Menacer Tidjani, René Lozi, Leon L. O. Chua
}

\section{To cite this version:}

Menacer Tidjani, René Lozi, Leon L. O. Chua. Hidden bifurcation in the Multispiral Chua Attractor. International journal of bifurcation and chaos in applied sciences and engineering , 2016, 26 (14), pp.1630039-1 1630023-26. 10.1142/S0218127416300391 . hal-01488550

\section{HAL Id: hal-01488550 https://hal.science/hal-01488550}

Submitted on 13 Mar 2017

HAL is a multi-disciplinary open access archive for the deposit and dissemination of scientific research documents, whether they are published or not. The documents may come from teaching and research institutions in France or abroad, or from public or private research centers.
L'archive ouverte pluridisciplinaire HAL, est destinée au dépôt et à la diffusion de documents scientifiques de niveau recherche, publiés ou non, émanant des établissements d'enseignement et de recherche français ou étrangers, des laboratoires publics ou privés. 


\title{
Hidden Bifurcations in the Multispiral Chua Attractor
}

\author{
Tidjani Menacer \\ Department of Mathematics, \\ University Mohamed Khider, Biskra, Algeria \\ tidjanimenacer@yahoo.fr \\ René Lozi \\ Université Côte d'Azur, CNRS, LJAD, \\ Parc Valrose 06108, Nice Cédex 02, France \\ rlozi@unice.fr \\ Leon O. Chua \\ Department of Electrical Engineering and Computer Sciences, \\ University of California, Berkeley, CA 94720, USA \\ chua@eecs.berkeley.edu
}

Received August 20, 2016

\begin{abstract}
We introduce a novel method revealing hidden bifurcations in the multispiral Chua attractor in the case where the parameter of bifurcation $c$ which determines the number of spiral is discrete. This method is based on the core idea of the genuine Leonov and Kuznetsov method for searching hidden attractors (i.e. applying homotopy and numerical continuation) but used in a very different way. Such hidden bifurcations are governed by a homotopy parameter $\varepsilon$ whereas $c$ is maintained constant. This additional parameter which is absent from the initial problem is perfectly fitted to unfold the actual structure of the multispiral attractor. We study completely the multispiral Chua attractor, generated via sine function, and check numerically our method for odd and even values of $c$ from 1 to 12 . In addition, we compare the shape of the attractors obtained for the same value of parameter $\varepsilon$ while varying the parameter $c$.
\end{abstract}

Keywords: Chua circuit; multispiral attractors; hidden bifurcations.

\section{Introduction}

The study of changes in the qualitative or topological structure of a given family of solutions of differential equations which depends on a continuous parameter is known as bifurcation theory [Sattinger, 1973; Lozi, 1975]. Initially the name "point of bifurcation" was defined as the value of the parameter for which the number of solutions of such a family of differential equations is increased from one to two, like the bifurcation of a branch of a tree, or when a road divides suddenly in two different ways. Nowadays bifurcation is understood as a small smooth change made to the parameter values (the bifurcation parameters) of a system of differential equations which causes a sudden "qualitative" or topological change in its behavior. However, when a system depends on a discrete parameter, no bifurcation can occur. In this article we overcome this dilemma by, showing that in some cases, "hidden bifurcation" instead of conventional bifurcation arises from a discrete parameter.

\subsection{The Chua attractor}

Fifty years ago, the discovery of the first chaotic strange attractor by E. N. Lorenz [Lorenz, 1963], introduced a drastic change in the mathematical study of oscillations. Fifty years after, only few chaotic attractors involving differential equations 
remain actively explored. Among them Chua attractor is nowadays incredibly used, because of both its realizations: electronic circuit or system of differential equations can be combined for multiple purposes [Duan et al., 2004]. Chua attractor is the asymptotic attractor of solutions of the system of differential equations modeling the dynamics of the Chua circuit. The Chua circuit is the simplest electronic circuit exhibiting chaos, and moreover possesses a very rich dynamical behavior which was verified from numerous laboratory experiments [Zhong \& Ayrom, 1985], computer simulations [Matsumoto, 1984], and rigorous mathematical analysis [Chua et al., 1986; Lozi \& Ushiki, 1993]. L. Chua invented it in the fall of 1983 [Chua, 1992] when he was visiting T. Matsumoto at Waseda University (Tokyo, Japan). This electronic circuit was mimicking directly on an oscilloscope screen a chaotic signal. Its invention was due in response to the search of two unfulfilled quests among many researchers on chaos concerning two wanting aspects of the Lorenz equations. The first quest was to demonstrate chaos as a robust physical phenomenon, and not merely an artifact of computer round-off errors. The second one was to prove that a strange attractor, which was obtained by computer simulation, is indeed chaotic in a rigorous mathematical sense. This circuit was the first real (concrete) example of a physical device exhibiting nonperiodic oscillations.

Since that discovery the Chua circuit has been generalized to several versions. One generalization substitutes the continuous piecewise-linear function by a smooth function, such as a cubic polynomial [Khibnik et al., 1993; Shilnikov, 1994; Huang et al., 1996; Hirsch et al., 2003], etc.

Complex attractors with $n$-double spirals were reported in [Suykens \& Vandewalle, 1991] by introducing additional break points in the nonlinear element in the Chua circuit, or using cellular neural networks with a piecewise-linear output function [Arena et al., 1996]. It is also demonstrated in [Suykens et al., 1997; Yalçin et al., 1999], that odd number scrolls can be observed by a similar modification. In [Tang et al., 2001], it is shown that the $n$-spirals attractors can be obtained with a simple sine or a cosine function.

\subsection{Hidden attractors}

Recently a new concept concerning the classification of attractors has been introduced: periodic or chaotic attractors belong either to the class of selfexcited attractors or to the class of hidden attractors [Leonov et al., 2011; Leonov \& Kuznetsov, 2011]. The basin of attraction of a self-excited attractor overlaps with the neighborhood of an equilibrium point, therefore self-excited attractors are very easy to be found. On the contrary a hidden attractor has a basin of attraction that does not intersect with small neighborhoods of any equilibrium points thereby making it very difficult to find; it is why one can call it hidden. Hidden attractors are important in engineering applications because they allow unexpected and potentially disastrous responses to perturbations in a structure like an aircraft's control systems (windup and antiwindup), drilling systems and electrical machines and in secure (chaotic) communications. In [Leonov, 2009b; Leonov et al., 2010], N. V. Kuznetsov and G. A. Leonov have proposed an effective method for the numerical localization of hidden attractors in multidimensional dynamical systems. This method is based on homotopy and numerical continuation. They construct a sequence of similar systems such that for the first (starting) system the initial data for numerical computation of oscillating solution (starting oscillation) can be obtained analytically. Then the transformation of this starting solution is tracked numerically in passing from one system to another. The first example of a hidden chaotic strange attractor was found in the Chua attractor [Leonov et al., 2012].

\subsection{Hidden bifurcations}

As mentioned before, because the parameter $c$ governing the number of spirals in the modified Chua circuit with sine function [Tang et al., 2001] is an integer, it is not possible to vary it continuously and therefore it is not possible to observe bifurcation of attractors from $n$ to $n+2$ spirals when the parameter $c$ changes. Moreover it is not possible to use noninteger real values for $c$. To overcome this obstacle, we use the core idea of the Leonov and Kuznetsov method for searching hidden attractors (i.e. homotopy and numerical continuation) in a different way, in order to uncover hidden bifurcations, governed by a homotopy parameter $\varepsilon$ while keeping $c$ constant.

This paper is organized as follows: In Sec. 2, we recall the model of Chua's system with multiplespiral attractors generated via sine function as proposed in [Tang et al., 2001]. In Sec. 3, we present 
the analytical-numerical method for hidden attractor localization proposed by Leonov [2009a, 2010]. The third section is devoted to the localization of hidden bifurcation in Chua's system with multiplespiral attractors. In addition, the results of numerical bifurcation of the analysis are given in Sec. 4 . Finally, the conclusion with some discussion is given in Sec. 5.

\section{Chua's System with Multiple-Spiral Attractors Generated via the Sine Function}

In recent years, various approaches have been proposed, for generating $n$-scroll in Chua's circuit. In [Tang et al., 2001], a new family of continuous functions for generating $n$-scroll attractors is proposed. It is shown that $n$-scroll attractors can be obtained with a simple sine or cosine function. Their approach allows us to design $n$-scroll attractors by modifying only two parameters in the function. The dimensionless state equation of Chua circuit modified with a sine function is given by:

$$
\left\{\begin{array}{l}
\dot{x}=\alpha(y-f(x)) \\
\dot{y}=x-y+z \\
\dot{z}=\beta z
\end{array}\right.
$$

where

$$
f(x)= \begin{cases}\frac{b \pi}{2 a}(x-2 a c), & \text { if } x \geq 2 a c \\ -b \sin \left(\frac{\pi x}{2 a}+d\right), & \text { if }-2 a c<x<2 a c \\ \frac{b \pi}{2 a}(x+2 a c), & \text { if } x \leq-2 a c\end{cases}
$$

where, $\alpha, \beta, a, b$ and $d$ are parameters belonging to $\mathbb{R}$ to be specified below for different applications, while $c$ is an integer. In this article, we call $n$-spirals such attractors instead of $n$-scroll, in order to avoid any confusion with the genuine work of Chua in which the inner structure of the Chua attractor is one scroll embedded in another scroll visible in the Poincaré section. In this article we are not interested in the fractal structure of the attractors but only in their general shape described in terms of the number of spirals.

An $n$-spirals attractor is generated when

$$
n=c+1,
$$

where $c$ and $n \in \mathbb{N}$ and

$$
d= \begin{cases}\pi, & \text { if } n \text { is odd } \\ 0, & \text { if } n \text { is even }\end{cases}
$$

It can be easily verified that $c$ governs the number of periods existing in the function, and henceforth the number of equilibrium points of (1). The equilibrium points are $\left(x_{\mathrm{eq}}, 0,-x_{\mathrm{eq}}\right)$ with $x_{\mathrm{eq}}=2 a k$ and $k=0, \pm 1, \ldots, \pm c$ [Tang et al., 2001].

When $\alpha=10.814, \beta=14.0, a=1.3, b=0.11$; 2-spirals, 4-spirals, 6-spirals, 8-spirals, 10-spirals and 12-spirals attractors are generated by Eqs. (1) and (2) for odd values of parameter $c: c=1, c=3$, $c=5, c=7, c=9$ and $c=11$, respectively. In the case of even values of $c: c=2, c=4$, $c=6, c=8, c=10$ and $c=12$, respectively, one obtains odd number of spirals: 3-spirals, 5spirals, 7-spirals, 9-spirals, 11-spirals and 13-spirals attractors, as depicted in Figs. 1(a)-1(f) and in Figs. 2(a)-2(f).

\section{Analytical-Numerical Method for Localization of Hidden Attractors}

In their articles [Leonov, 2009b; Leonov et al., 2010], N. V. Kuznetsov and G. A. Leonov have proposed an effective method for the numerical localization of hidden attractors in multidimensional dynamical systems. This method is based on homotopy and numerical continuation. They construct a sequence of similar systems such that for the first (starting) system the initial data for numerical computation of an oscillating solution (starting oscillation) can be obtained analytically. Then the transformation of this starting solution is tracked numerically in passing from one system to another. This proposed approach is generalized in the work [Leonov \& Kuznetsov, 2011] to the systems of the form

$$
\frac{d X}{d t}=P X+\psi(X)
$$

where $P$ is a constant $n \times n$-matrix, $\psi(X)$ is a continuous vector-function, and $\psi(0)=0$.

In the case where there is only one scalar nonlinearity in the system, it takes the following form

$$
\frac{d X}{d t}=P X+q \psi\left(r^{t} X\right), \quad X \in \mathbb{R}^{n} .
$$

Here $q, r$ are constant $n$-dimensional vectors, $t$ is a transposition operation, $\psi(\sigma)$ is a continuous 

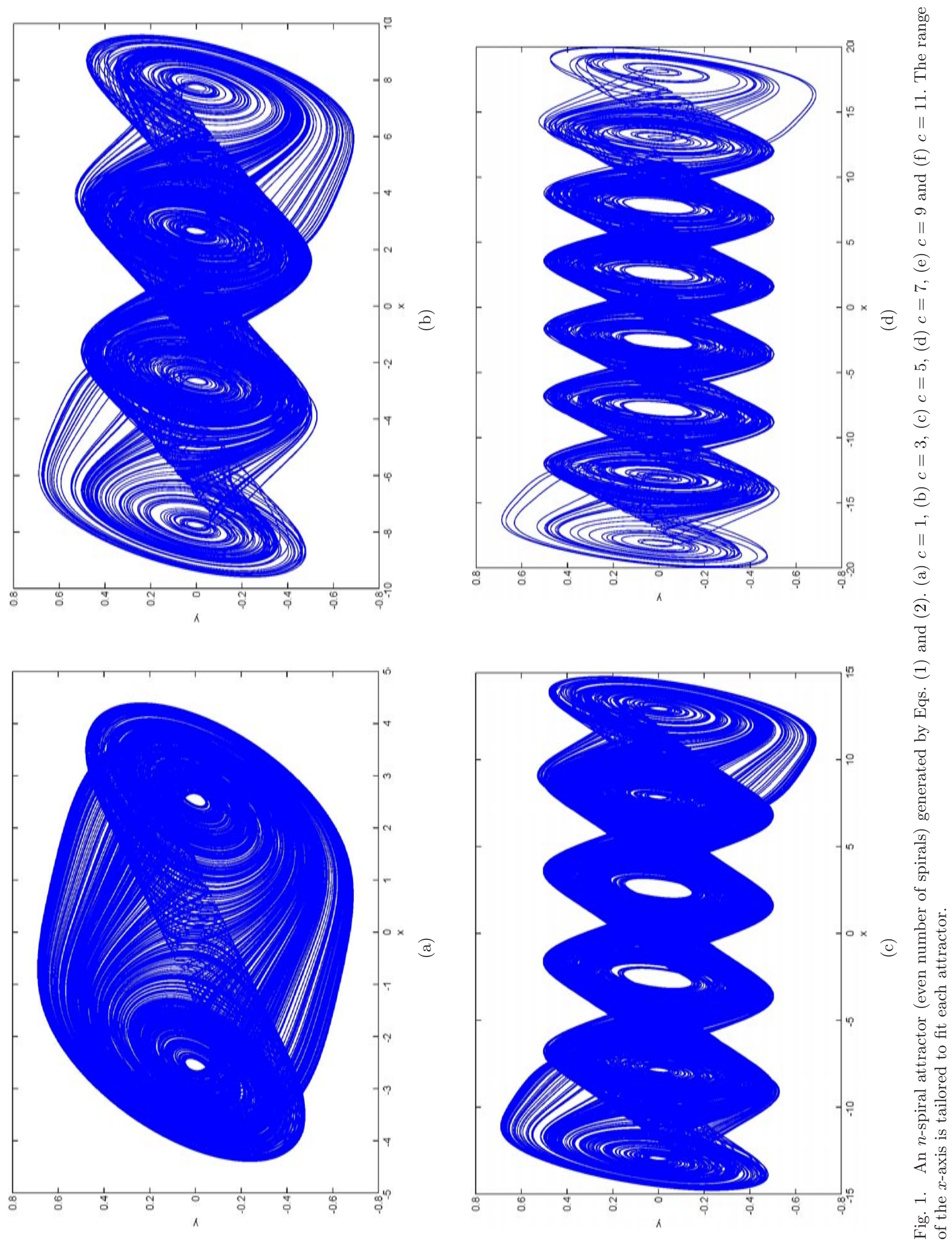

page 4 

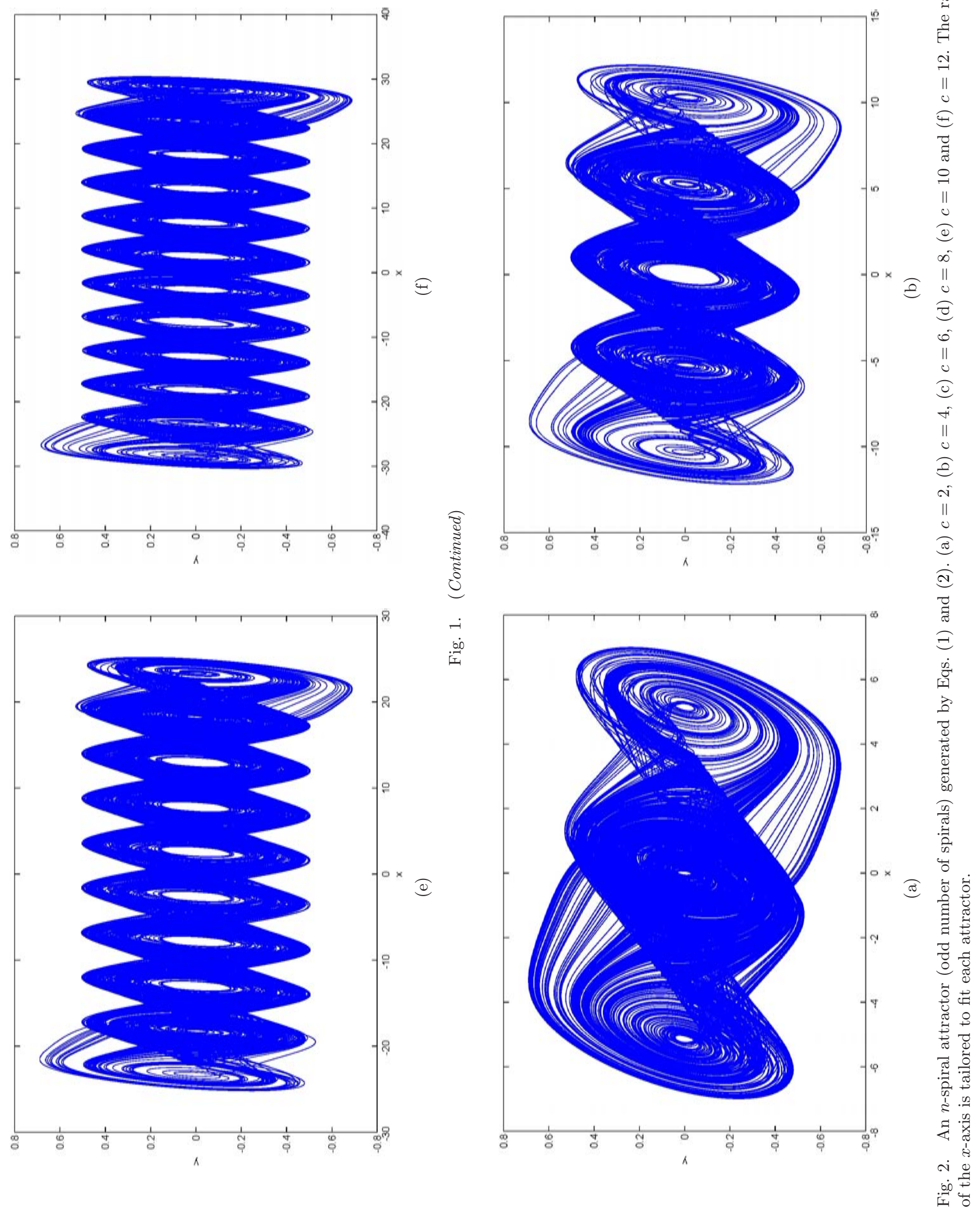

page 5 

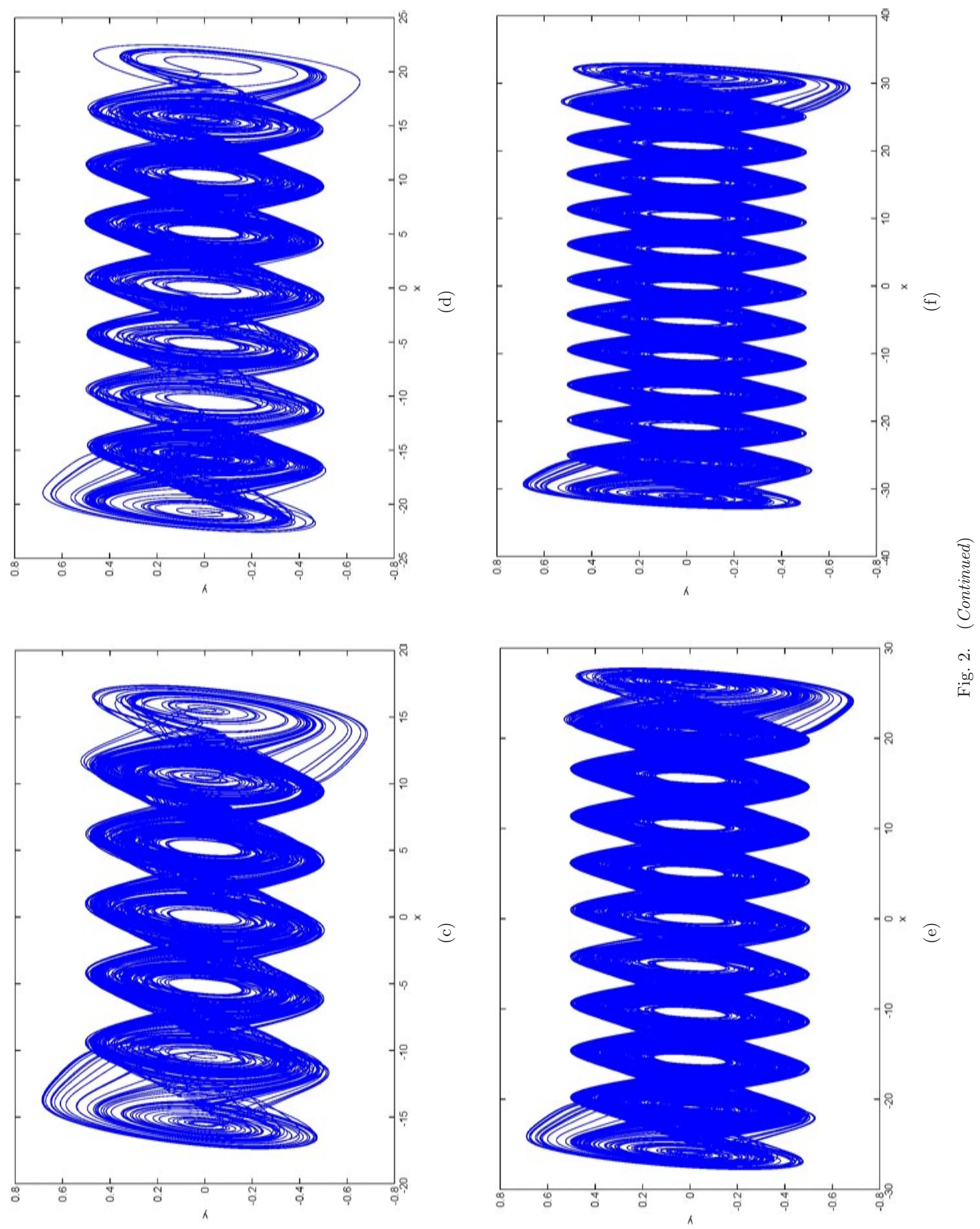

page 6 
piecewise-differentiable scalar function satisfying the condition $\psi(0)=0$. Now let us define a coefficient of harmonic linearization $k$ in such a way that the matrix

$$
P_{0}=P+k q r^{t}
$$

has a pair of purely imaginary eigenvalues $\pm i \omega_{0}$, $\left(\omega_{0}>0\right)$ and the rest of its eigenvalues have negative real parts. We assume that such $k$ exists and we rewrite the system (6) as

$$
\frac{d X}{d t}=P_{0} X+q \varphi\left(r^{t} X\right)
$$

where $\varphi(\tau)=\psi(\tau)-k \tau$.

Now we introduce a finite sequence of functions $\varphi^{0}(\tau), \varphi^{1}(\tau), \ldots, \varphi^{m}(\tau)$ such that the graph of the functions $\varphi^{j}(\tau)$ and $\varphi^{j+1}(\tau),(j=0, \ldots, m-1)$, differ slightly from one another, where the function $\varphi^{0}(\tau)$ is small and $\varphi^{m}(\tau)=\varphi(\tau)$. Using a smallness of function, we can apply the method of harmonic linearization (describing function method) for the system

$$
\frac{d X}{d t}=P_{0} X+q \varphi^{0}\left(r^{t} X\right)
$$

in order to determine the nontrivial stable periodic solution $X^{0}(t)$. For the localization of the attractor of the original system (8), we will follow numerically the transformation of this periodic solution (a starting oscillating attractor, not including equilibria, denoted further by $A_{0}$ with increasing $j$. To determine the initial condition $X^{0}(0)$ of the periodic solution, system (9) can be transformed by a linear nonsingular transformation $S(X=S Y)$ to the form:

$$
\left\{\begin{array}{l}
\dot{y}_{1}=-\omega_{0} y_{2}+b_{1} \varphi^{0}\left(y_{1}+c_{3}^{t} Y_{3}\right) \\
\dot{y}_{2}=\omega_{0} y_{1}+b_{2} \varphi^{0}\left(y_{1}+c_{3}^{t} Y_{3}\right) \\
\dot{Y}_{3}=A_{3} Y_{3}+B_{3} \varphi^{0}\left(y_{1}+c_{3}^{t} Y_{3}\right)
\end{array}\right.
$$

Here $y_{1}, y_{2}$ are scalar values, $Y_{3}$ is an $(n-2)$ dimensional vector; $B_{3}$ and $c_{3}$ are $(n-2)$ dimensional vectors, $b_{1}$ and $b_{2}$ are real numbers; $A_{3}$ is an $(n-2) \times(n-2)$ matrix, where all of its eigenvalues have negative real parts. Without loss of generality, it can be assumed that for the matrix $A_{3}$ there exists a positive number $d_{1}>0$ such that

$$
Y_{3}^{t}\left(A_{3}+A_{3}^{t}\right) Y_{3} \leq-2 d_{1}\left|Y_{3}^{2}\right|, \quad \forall Y_{3} \in \mathbb{R}^{n-2} .
$$

In the scalar case, let us define the following describing function $\Phi$ of a real variable $\tau$, and assume the existence of its derivative:

$$
\Phi(\tau)=\int_{0}^{2 \pi / \omega_{0}} \varphi\left(\cos \left(\omega_{0} t\right) \tau\right) \cos \left(\omega_{0} t\right) d t .
$$

Theorem 1 [Leonov et al., 2010]. If a positive $\tau_{0}$ can be found such that

$$
\Phi\left(\tau_{0}\right)=0,\left.\quad b_{1} \frac{d \Phi(\tau)}{d \tau}\right|_{\tau=\tau_{0}}<0,
$$

then for the initial condition of the periodic solution $X^{0}(0)=S\left(y_{1}(0), y_{2}(0), Y_{3}(0)\right)^{T}$ at the first step of algorithm we have $y_{1}(0)=\tau_{0}+O(\varepsilon), y_{2}(0)=$ $0, Y_{3}(0)=O_{n-2}(\varepsilon)$, where $O_{n-2}(\varepsilon)$ is an $(n-2)$ dimensional vector such that all its components are $O(\varepsilon)$.

For the stability of $x^{0}(t)$ (where stability is defined in the sense that for all solutions with the initial data sufficiently close to $x^{0}(0)$ the modulus of their difference with $x^{0}(t)$ is uniformly bounded for all $t>0$ ), it is sufficient to require the following condition is true

$$
\left.b_{1} \frac{d \Phi(\tau)}{d \tau}\right|_{\tau=\tau_{0}}<0
$$

In practice, to determine $k$ and $\omega_{0}$ one uses the transfer function $W(p)$ of system (8)

$$
W(p)=r(P-p I)^{-1} q
$$

where $p$ is a complex variable. The number $\omega_{0}$ is determined from the equation $\operatorname{Im} W\left(i \omega_{0}\right)=0$ and $k$ is calculated then by the formula $k=$ $-\operatorname{Re} W\left(i \omega_{0}\right)^{-1}$.

\section{Localization of Hidden Bifurcation in Chua's System with Multiple Spiral Attractors Generated via the Sine Function}

Theorem 1 can be used for the search of hidden attractors in Chua's system with multiple spiral attractors generated via the sine function. Consider the given Chua's system (1) and (2). For this purpose, we rewrite system (1)-(2) into the form (5)

$$
\frac{d X}{d t}=P X+q \psi\left(r^{t} X\right), \quad X \in \mathbb{R}^{3},
$$


where

$$
\begin{gathered}
P=\left(\begin{array}{ccc}
0 & \alpha & 0 \\
1 & -1 & 1 \\
0 & -\beta & 0
\end{array}\right), \quad q=\left(\begin{array}{l}
\alpha \\
0 \\
0
\end{array}\right) \text { and } r=\left(\begin{array}{l}
1 \\
0 \\
0
\end{array}\right) \\
\psi(\sigma)=f(\sigma) .
\end{gathered}
$$

Introducing the coefficient $k$ and a small parameter $\varepsilon$, system (14) can be transformed into the form of system (9) as follows:

$$
\frac{d X}{d t}=P_{0} X+q \varepsilon \varphi^{0}\left(r^{t} X\right), \quad X \in \mathbb{R}^{3}
$$

where

$$
\begin{aligned}
P_{0}=P+k q r^{t} & =\left(\begin{array}{ccc}
-\alpha k & \alpha & 0 \\
1 & -1 & 1 \\
0 & -\beta & 0
\end{array}\right) \\
\varphi(\sigma) & =\psi(\sigma)-k \tau=-\alpha f(\sigma)-k \tau
\end{aligned}
$$

The transfer function $W_{P_{0}}(p)$ of the system (15) is given by

$$
\begin{aligned}
W_{P_{0}}(p) & =r^{t}(P-p I)^{-1} \\
& =\alpha \frac{p^{2}+p+\beta}{p^{3}+p^{2}+(\beta-\alpha) p} .
\end{aligned}
$$

For the parameter value $\alpha=10.814, \beta=14$, using the formulas $\operatorname{Im} W\left(i \omega_{0}\right)=0$ and $k=$ $-\left(\operatorname{Re} W\left(i \omega_{0}\right)\right)^{-1}$, we calculated $\omega_{0}=1.8766$ and $k=0.031084$. Via the nonsingular linear transformation $X=S Y$ the system (15) is reduced to the form

$$
\frac{d Y}{d t}=A Y+B \varepsilon \varphi\left(C^{t} Y\right)
$$

where

$$
A=S^{-1} P_{0} S, \quad B=S^{-1} q \quad \text { and } \quad C^{t}=r^{t} S .
$$

This implies

$$
\begin{gathered}
A=\left(\begin{array}{ccc}
0 & -\omega_{0} & 0 \\
\omega_{0} & 0 & 0 \\
0 & 0 & -d
\end{array}\right), \quad B=\left(\begin{array}{l}
b_{1} \\
b_{2} \\
1
\end{array}\right), \\
C=\left(\begin{array}{c}
1 \\
0 \\
-h
\end{array}\right) \quad \text { and } \quad S=\left(\begin{array}{lll}
S_{11} & S_{12} & S_{13} \\
S_{21} & S_{22} & S_{23} \\
S_{31} & S_{32} & S_{33}
\end{array}\right) .
\end{gathered}
$$

The transfer function $W_{A}(p)$ of system (16) can be represented as

$$
\begin{aligned}
W_{A}(p) & =\frac{-b_{1} p+b_{2} \omega_{0}}{p^{2}+\omega_{0}^{2}}+\frac{h}{p+d} \\
& =\alpha \frac{p^{2}+p+\beta}{p \beta-p \alpha+p^{2}+p^{3}+k p \alpha+k \alpha \beta+k \alpha p^{2}} .
\end{aligned}
$$

Further, using the equality of transfer functions of systems (15) and (16), we obtain

$$
\begin{gathered}
k=\frac{\alpha+\omega_{0}^{2}-\beta}{\alpha}, \quad d=\alpha+\omega_{0}^{2}-\beta+1, \\
h=\frac{\alpha\left(\beta-d+d^{2)}\right.}{\omega_{0}^{2}+d^{2}}, \quad b_{1}=\frac{\alpha\left(\beta-\omega_{0}^{2}-d\right)}{\omega_{0}^{2}+d^{2}} \\
b_{2}=\frac{\alpha(1-d)\left(\omega_{0}+\beta d\right)}{\omega_{0}^{2}\left(\omega_{0}^{2}+d^{2}\right)} .
\end{gathered}
$$

When the parameters of system (14) are fixed at, $\alpha=10.814, \beta=14.0, a=1.3, b=0.11$, we obtain

$$
\begin{array}{r}
k=0.031071, \quad d=1.336, \quad h=29.443, \\
b_{1}=18.629, \quad b_{2}=-0.36483, \\
S=\left(\begin{array}{ccc}
1 & 0 & -29.443 \\
0.031084 & 0.17354 & 2.7223 \\
-1.2946 & 0.23188 & 34.917
\end{array}\right) .
\end{array}
$$

Via Theorem 1, for $\varepsilon$ small enough, we calculate the following initial condition for the first step of the multistage localization procedure

$$
X(0)=S Y(0)=S\left(\begin{array}{c}
\tau_{0} \\
0 \\
0
\end{array}\right)=\left(\begin{array}{c}
\tau_{0} S_{11} \\
\tau_{0} S_{21} \\
\tau_{0} S_{31}
\end{array}\right) .
$$

For the Chua multispiral model, the number of spirals is an integer determined uniquely by the value of parameter $c$, this number is $c+1$. Therefore $c$ being an integer it is not possible to do a continuous variation of this parameter in order to observe bifurcations from $p$ to $p+2$ spirals. The main idea of this article to overcome such obstacle is to introduce a continuous additional parameter $\varepsilon$ in order to uncover hidden bifurcations. We call such bifurcations hidden bifurcations because an additional parameter is needed to unfold the structure of the multispiral attractor. 


\section{Numerical Results}

Consider the system (1) and (2) with parameter values

$\alpha=10.814, \quad \beta=14.0, \quad a=1.3, \quad b=0.11$.

Now we apply the localization procedure described above to Chua's system (14) with multiple spiral attractors. For this purpose, we compute the following starting frequency $\omega_{0}$ and a coefficient of harmonic linearization $k$ :

$$
\omega_{0}=1.8767 \text { and } k=0.031084 .
$$

Then, we compute the solutions of system (15) with the nonlinearity $\varepsilon \varphi(x)=\varepsilon(\psi(x)-k x)$, by increasing sequentially $\varepsilon$ from the value $\varepsilon=0.1$ to $\varepsilon=1$ by starting at the beginning with the step 0.1 , and decreasing it down to 0.001 between $\varepsilon=0.8$ and $\varepsilon=1$. Using (17), we obtain the initial conditions, for each integer value of $c$ (Tables 1 and 2), for the first step of our multistage procedure for constructing the solutions.

If the stable periodic solution $X^{0}(t)$ (corresponding to a very small $\varepsilon$ ) near the harmonic one is determined, all the points of the stable periodic solution $X^{0}(t)$ are located in the domain of attraction of the stable periodic solution $X^{1}(t)$ of the system. The solution $X^{1}(t)$ can be found numerically by searching one trajectory of system (5) with $\varepsilon=0.1$ from the initial point $X^{0}(0)$. For the value
Table 1. Initial condition as a function of odd integer $c$.

\begin{tabular}{clll}
\hline Parameter $c$ & \multicolumn{1}{c}{$x_{0}$} & \multicolumn{1}{c}{$y_{0}$} & \multicolumn{1}{c}{$z_{0}$} \\
\hline 1 & 4.22 & 0.13117 & -5.4632 \\
3 & 12.09 & 0.37581 & -15.652 \\
5 & 20.02 & 0.6223 & -25.918 \\
7 & 28 & 0.87035 & -36.249 \\
9 & 36 & 1.119 & -46.606 \\
11 & 43.95 & 1.3661 & -56.898 \\
\hline
\end{tabular}

Table 2. Initial condition as a function of even integer $c$.

\begin{tabular}{cccc}
\hline Parameter $c$ & \multicolumn{1}{c}{$x_{0}$} & \multicolumn{1}{c}{$y_{0}$} & \multicolumn{1}{c}{$z_{0}$} \\
\hline 2 & 8.13 & 0.25271 & -10.525 \\
4 & 16.05 & 0.49890 & -20.778 \\
6 & 24 & 0.74602 & -31.07 \\
8 & 31.99 & 0.99438 & -41.414 \\
10 & 39.97 & 1.2424 & -51.745 \\
12 & 47.95 & 1.4905 & -62.076 \\
\hline
\end{tabular}

of the parameter $\varepsilon=0.1$, after a transient process, the computational procedure reaches the starting oscillation $X^{1}(t)$.

We continue by increasing the parameter " $j$ " and using the numerical procedure, we obtain the sequential transformation $X^{j}(t)$ for the original Chua system (1).

Without loss of generality, we illustrate the case for $c=12$ and apply the above procedure to obtain

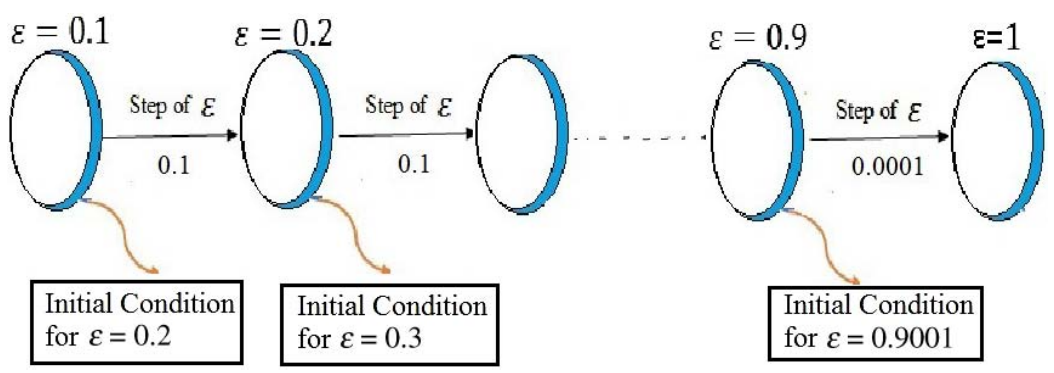

Fig. 3. Transition from one solution to another, from $\varepsilon=0.1$ to $\varepsilon=1$.

Table 3. Initial condition according to the values of $\varepsilon$.

\begin{tabular}{rrrrcrrr}
\hline$\varepsilon$ & \multicolumn{1}{c}{$x_{0}$} & \multicolumn{1}{c}{$y_{0}$} & \multicolumn{1}{c}{$x_{0}$} & \multicolumn{1}{c}{$y_{0}$} & \multicolumn{1}{c}{$z_{0}$} \\
\hline 0.1 & \multicolumn{1}{c}{47.95} & 1.4905 & -62.076 & 0.8221 & -2.1384 & -6.2713 & 2.6040 \\
0.2 & 1.2959 & 0.4052 & -1.1970 & 0.9718 & 1.6973 & 0.2080 & -2.2365 \\
0.3 & -1.0658 & 0.2227 & 1.9005 & 0.9952 & 2.1948 & 1.1740 & -2.7819 \\
0.4 & 2.2579 & 0.2227 & -2.7591 & 0.99 & -4.3244 & -0.3244 & -3.2898 \\
0.5 & -1.5593 & -0.3827 & 1.6249 & 0.995 & -0.9327 & -0.1236 & 1.3513 \\
0.6 & -2.4320 & -0.0300 & 3.1930 & 0.998 & -10.7179 & 0.1882 & 11.5265 \\
0.7 & -1.4814 & 0.3031 & 2.1874 & 0.999 & -2.6705 & 0.2845 & 2.2206 \\
0.8 & -2.7063 & -0.0658 & 3.3600 & 1 & -4.2597 & -0.1102 & 3.3563 \\
\hline
\end{tabular}


Table 4. Values of the parameter $\varepsilon$ at the bifurcation points for $c=12$ (13 spirals).

\begin{tabular}{lccccc}
\hline \multicolumn{5}{c}{$\varepsilon$} \\
\hline 0.8221 & 0.9718 & 0.9852 & 0.99 & 0.995 & 0.998 \\
3 spirals & 5 spirals & 7 spirals & 9 spirals & 11 spirals & 13 spirals \\
\hline
\end{tabular}

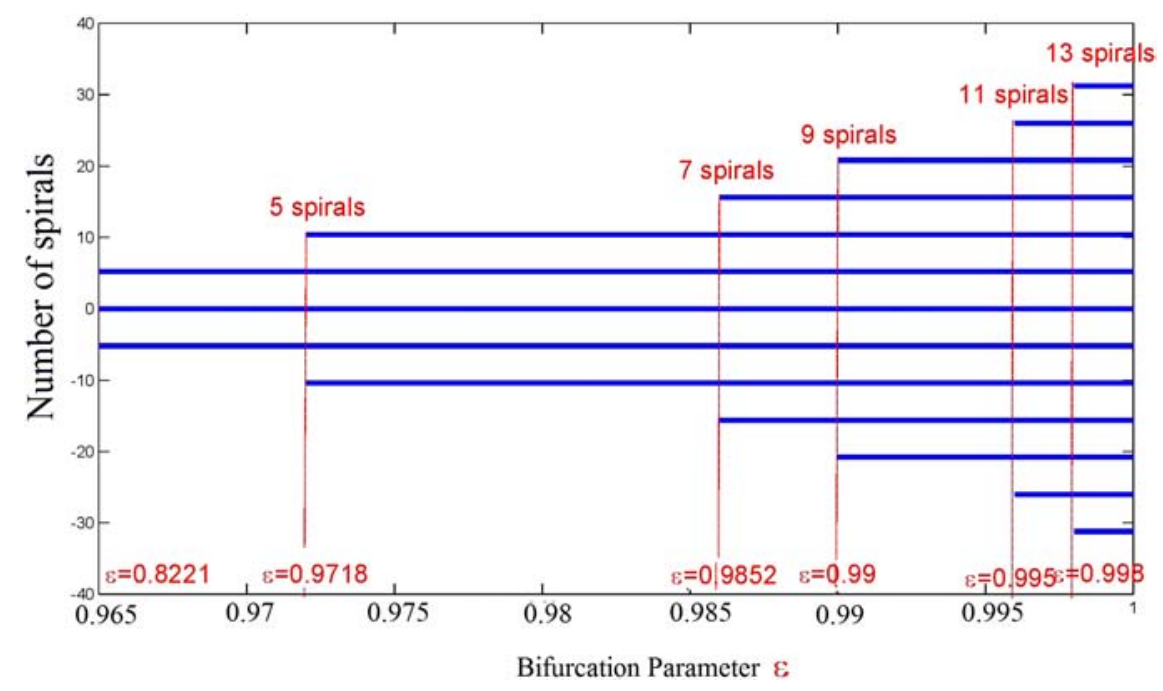

Fig. 4. Diagram of bifurcation.

the initial conditions for the solutions for increasing values of $\varepsilon$, as shown in Fig. 3 .

Using this initial condition we get at the points of bifurcation, where the attractor changes the number of spirals from one to 13 spirals as shown in Table 4 and in Figs. 4-6. It should be noted that the number of bifurcation points is independent of the parameter $\varepsilon$ (it depends only on the parameter $c$ ). So the role of the parameter $\varepsilon$ is to increase the number of spirals of the attractor after the bifurcation point. Figure 4 shows the bifurcation diagram (i.e. the change of number of spirals from $n$ to $n+2, n=1, \ldots, 11)$. Table 5 presents the bifurcation points for different values of the parameter $c$

Table 5. Bifurcations points for different values of parameter $c$.

\begin{tabular}{|c|c|c|c|c|c|c|}
\hline$c$ & 2 Spirals & 4 Spirals & 6 Spirals & 8 Spirals & 10 Spirals & 12 Spirals \\
\hline 1 & 0.8535 & impossible & impossible & impossible & impossible & impossible \\
\hline 3 & 0.91625 & 0.9491127 & impossible & impossible & impossible & impossible \\
\hline 5 & 0.9161999 & 0.94915 & 0.97979 & impossible & impossible & impossible \\
\hline 7 & 0.9161999 & 0.94915 & 0.9797501 & 0.9889 & impossible & impossible \\
\hline 9 & 0.9161999 & 0.94915 & 0.97979 & 0.9888989 & 0.99404901 & impossible \\
\hline 11 & 0.9161999 & 0.9491125 & 0.9797505 & 0.9888989 & 0.99404901 & 0.998950169 \\
\hline$c$ & 3 Spirals & 5 Spirals & 7 Spirals & 9 Spirals & 11 Spirals & 13 Spirals \\
\hline 2 & 0.8221 & impossible & impossible & impossible & impossible & impossible \\
\hline 4 & 0.8221 & 0.9718 & impossible & impossible & impossible & impossible \\
\hline 6 & 0.8221 & 0.9718 & 0.9852 & impossible & impossible & impossible \\
\hline 8 & 0.8221 & 0.9718 & 0.9852 & 0.99 & impossible & impossible \\
\hline 10 & 0.8221 & 0.9718 & 0.9852 & 0.99 & 0.999 & impossible \\
\hline 12 & 0.8221 & 0.9718 & 0.9852 & 0.99 & 0.995 & 0.998 \\
\hline
\end{tabular}


(a)

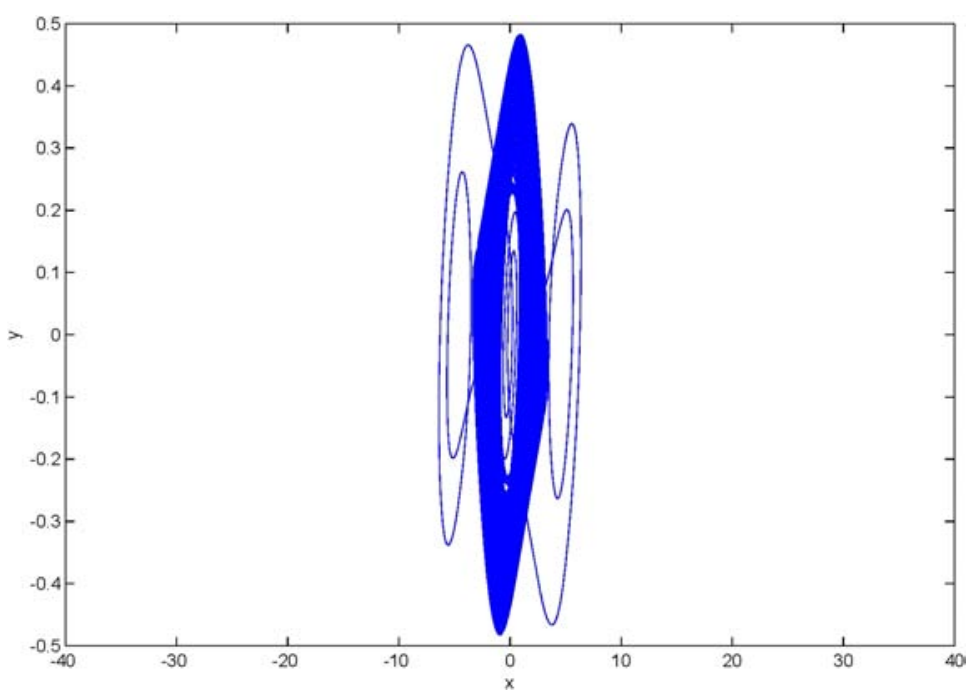

(b)

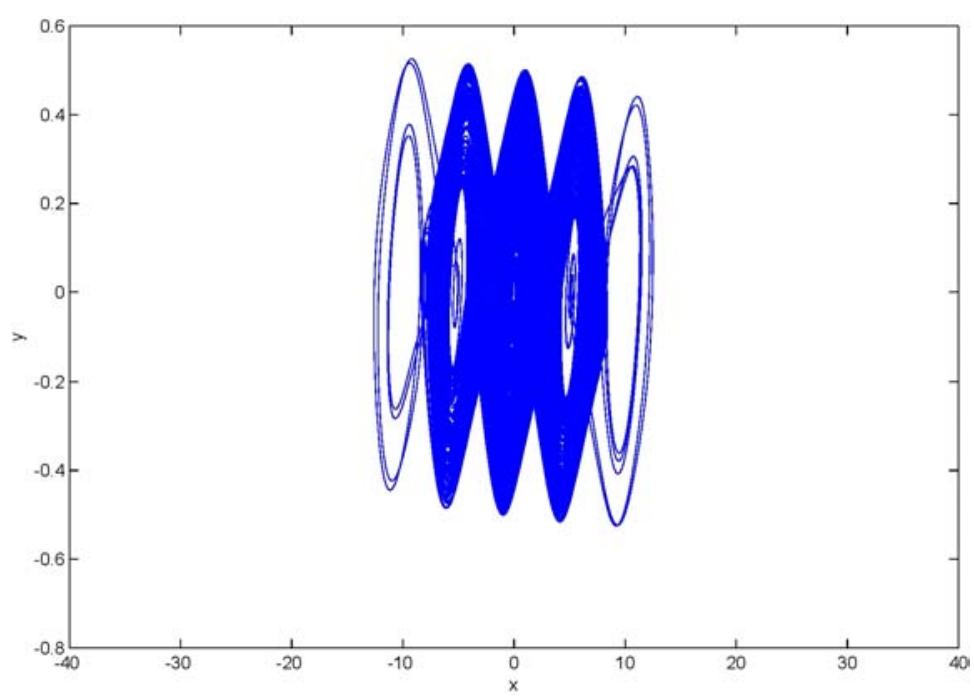

(c)

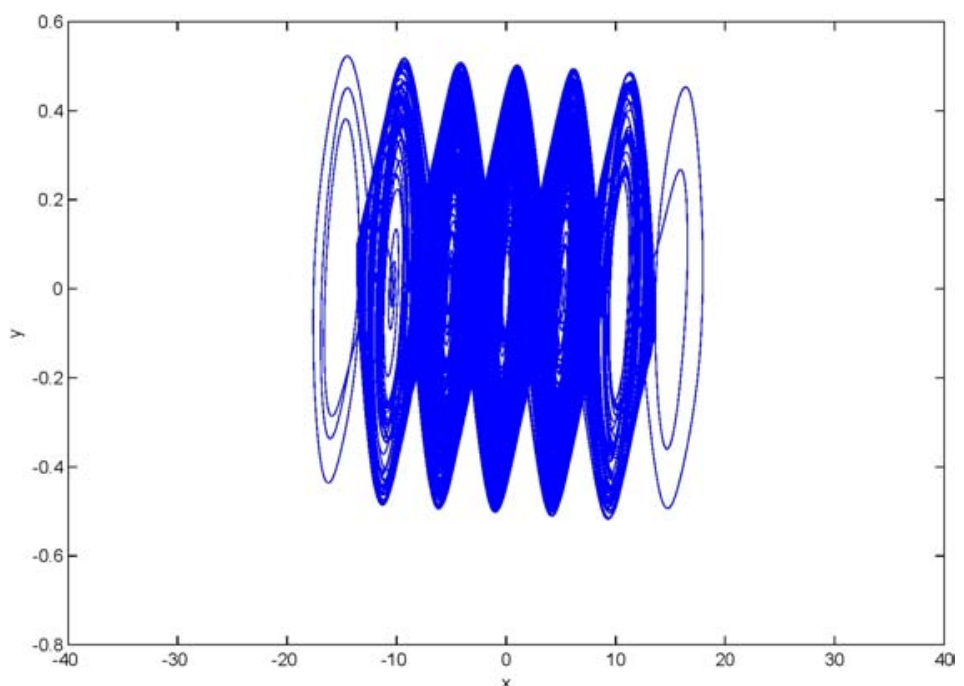

Fig. 5. The increasing number of spirals of the system (15) according to increasing $\varepsilon$ values. (a) 3 spirals for $c=12$ and $\varepsilon=0.8221$, (b) 5 spirals for $c=12$ and $\varepsilon=0.9718$ and (c) 7 spirals for $c=12$ and $\varepsilon=0.9852$. The range of the $x$-axis is the same for Figs. 5-12. 
(a)

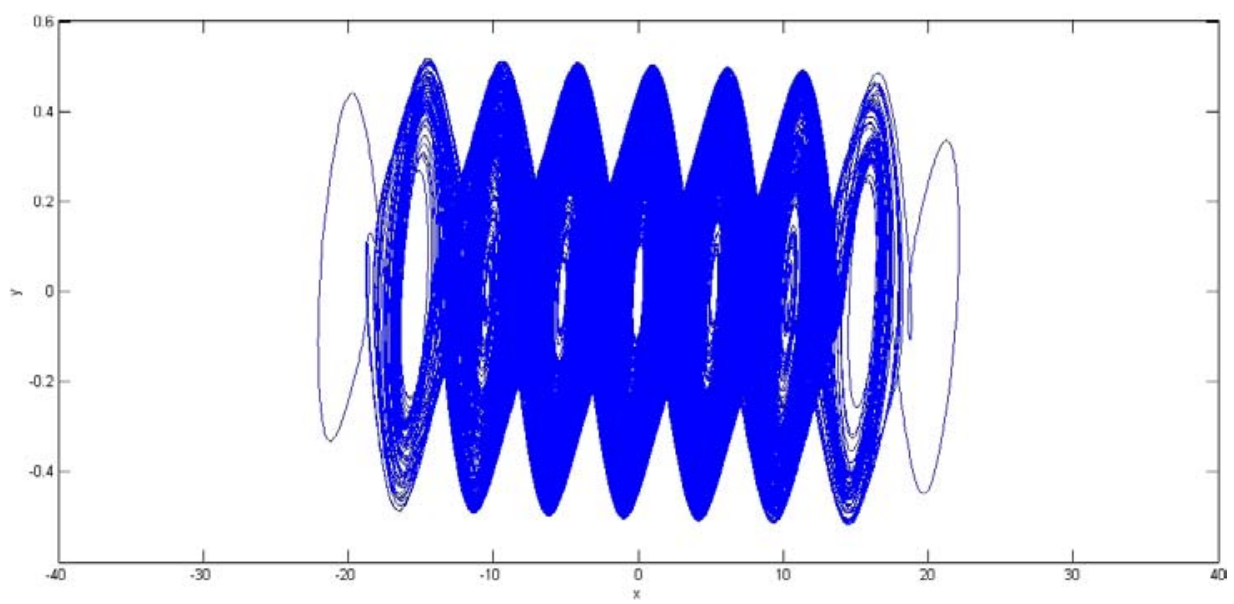

(b)

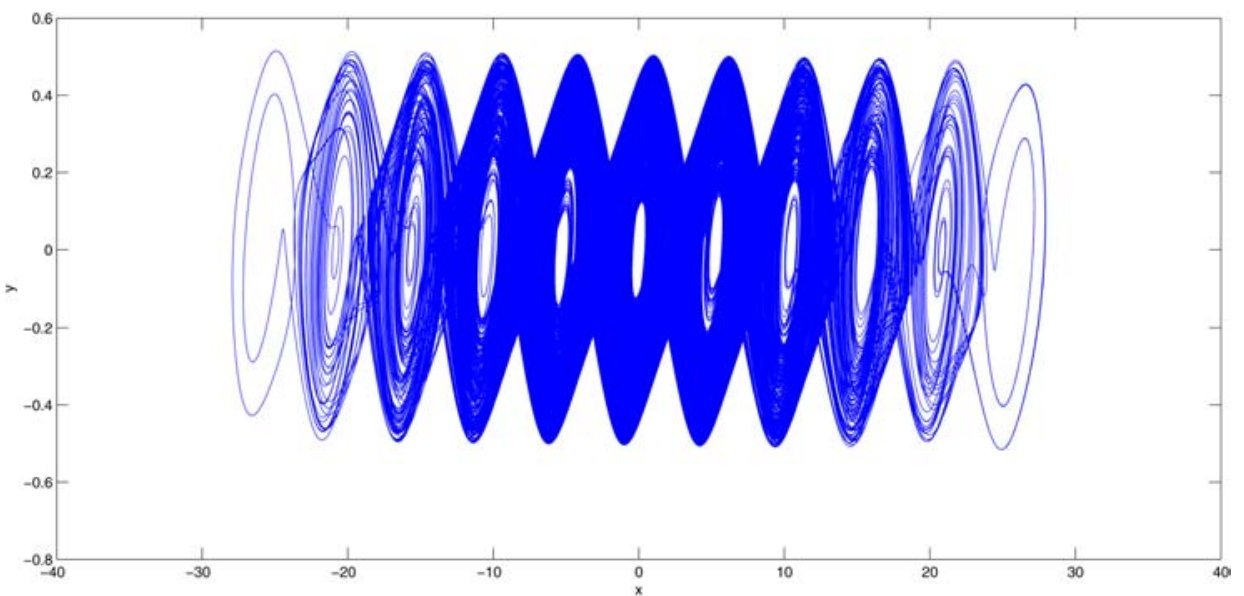

(c)

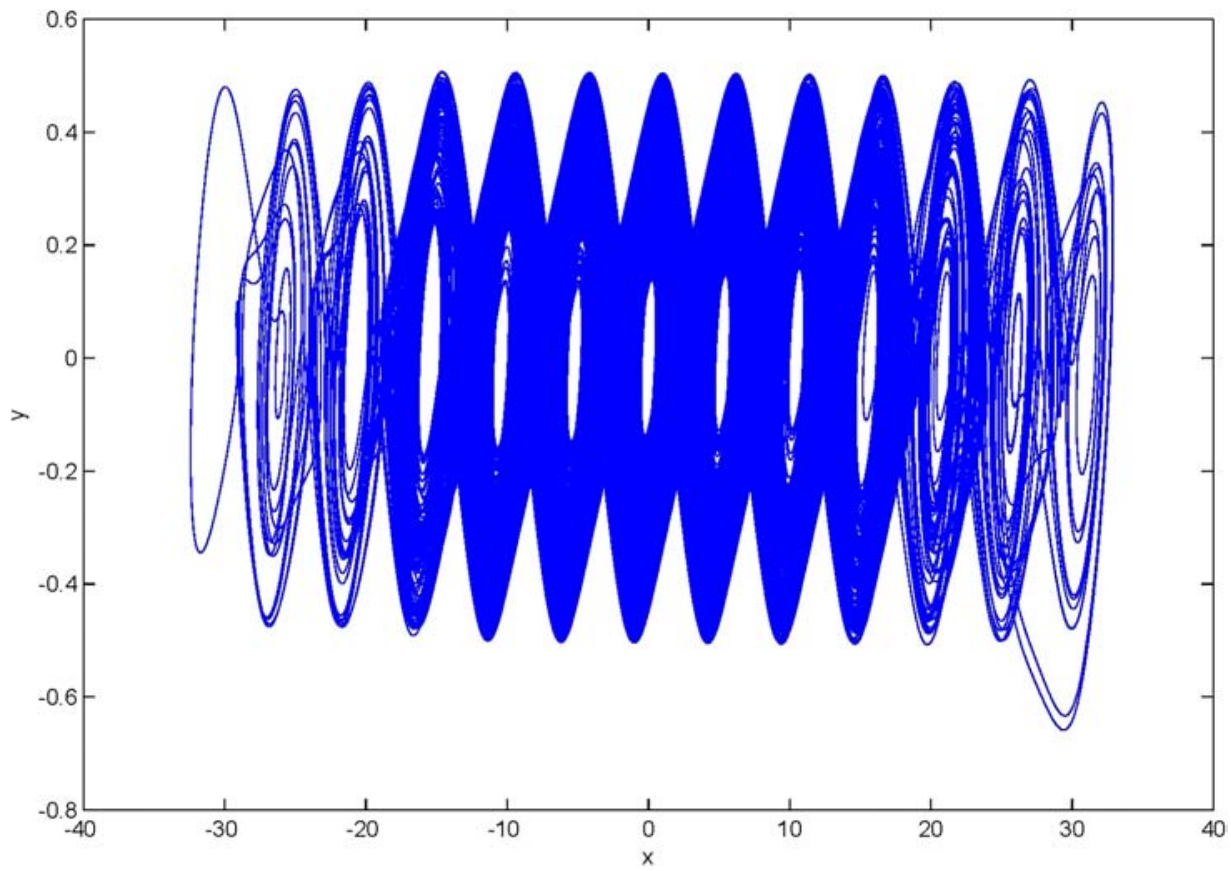

Fig. 6. The increasing number of spirals of the system (15) according to increasing $\varepsilon$ values. (a) 9 spirals for $c=12$ and $\varepsilon=0.99$, (b) 11 spirals for $c=12$ and $\varepsilon=0.995$ and (c) 13 spirals for $c=12$ and $\varepsilon=0.998$. The range of the $x$-axis is the same for Figs. $5-12$. 
(a)

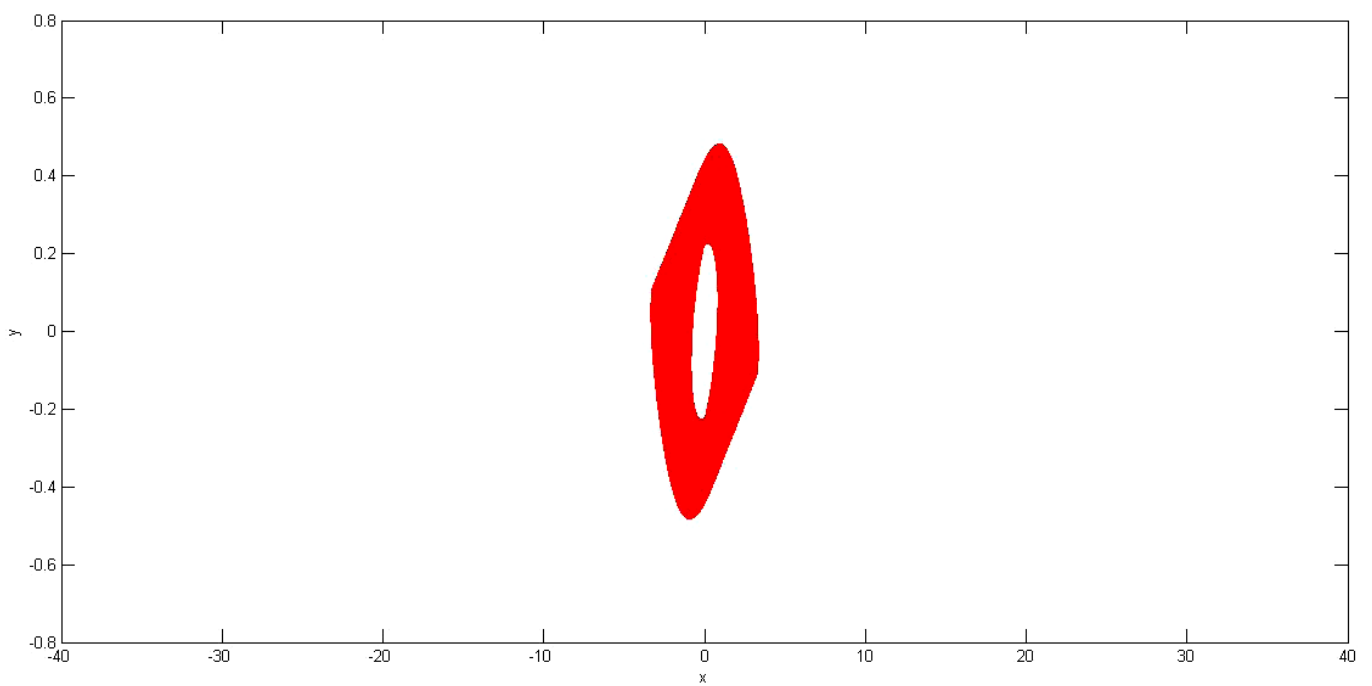

(b)

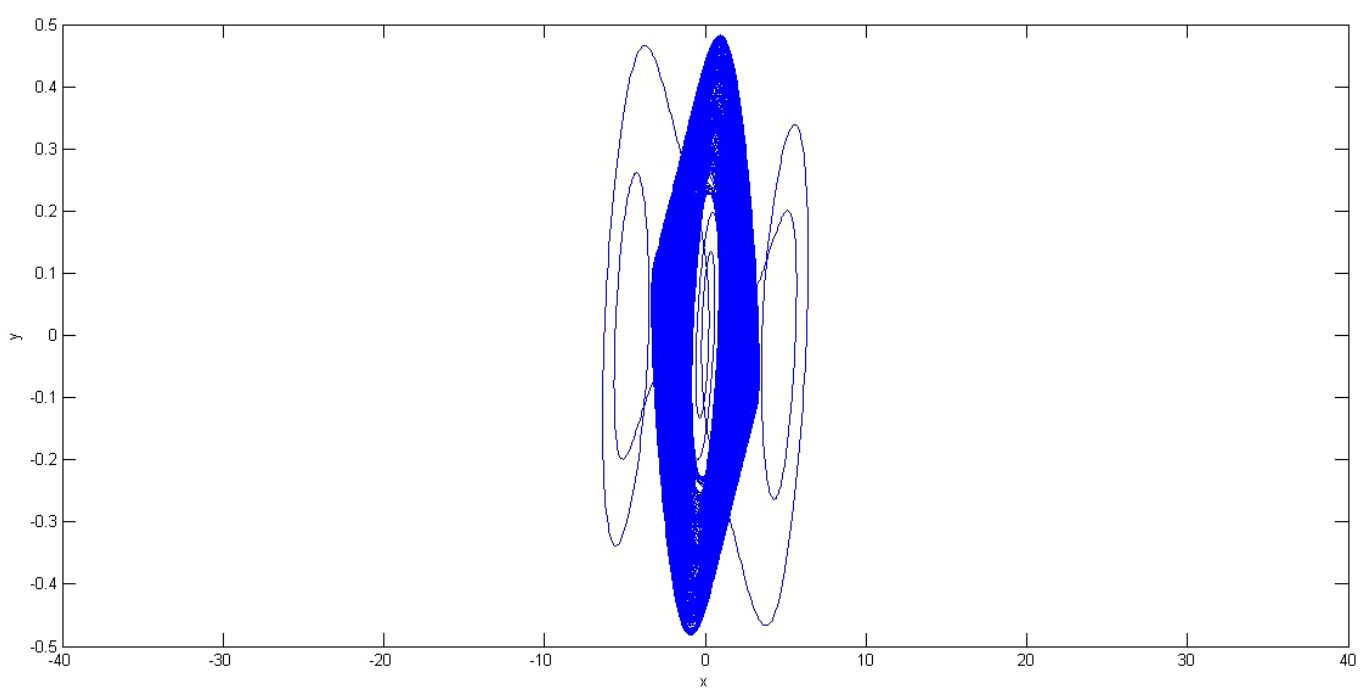

(c)

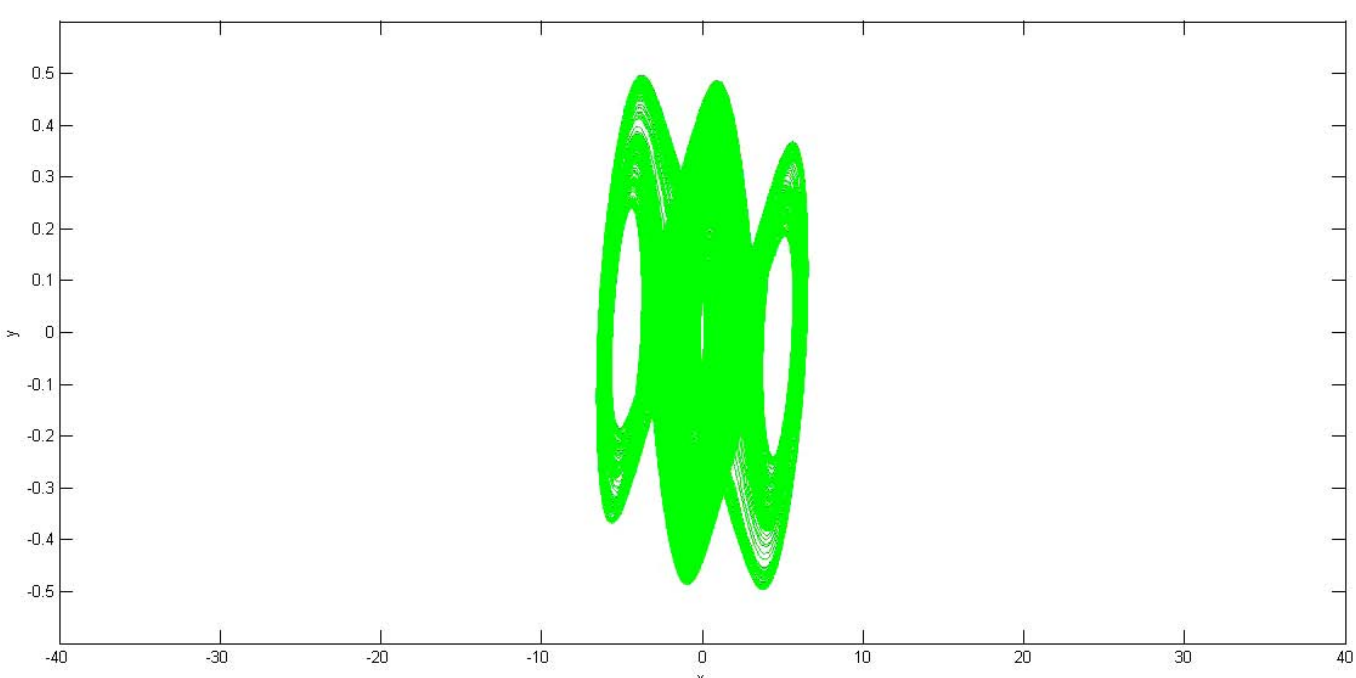

Fig. 7. $n$-scroll (odd scroll) attractors generated by the hidden bifurcation method for $c=2$. (a) 1 spiral for $\varepsilon=0.82$, (b) 3 spirals for $\varepsilon=0.8221$ and (c) 3 spirals for $\varepsilon=0.85$. The range of the $x$-axis is the same for Figs. $5-12$. 
(a)

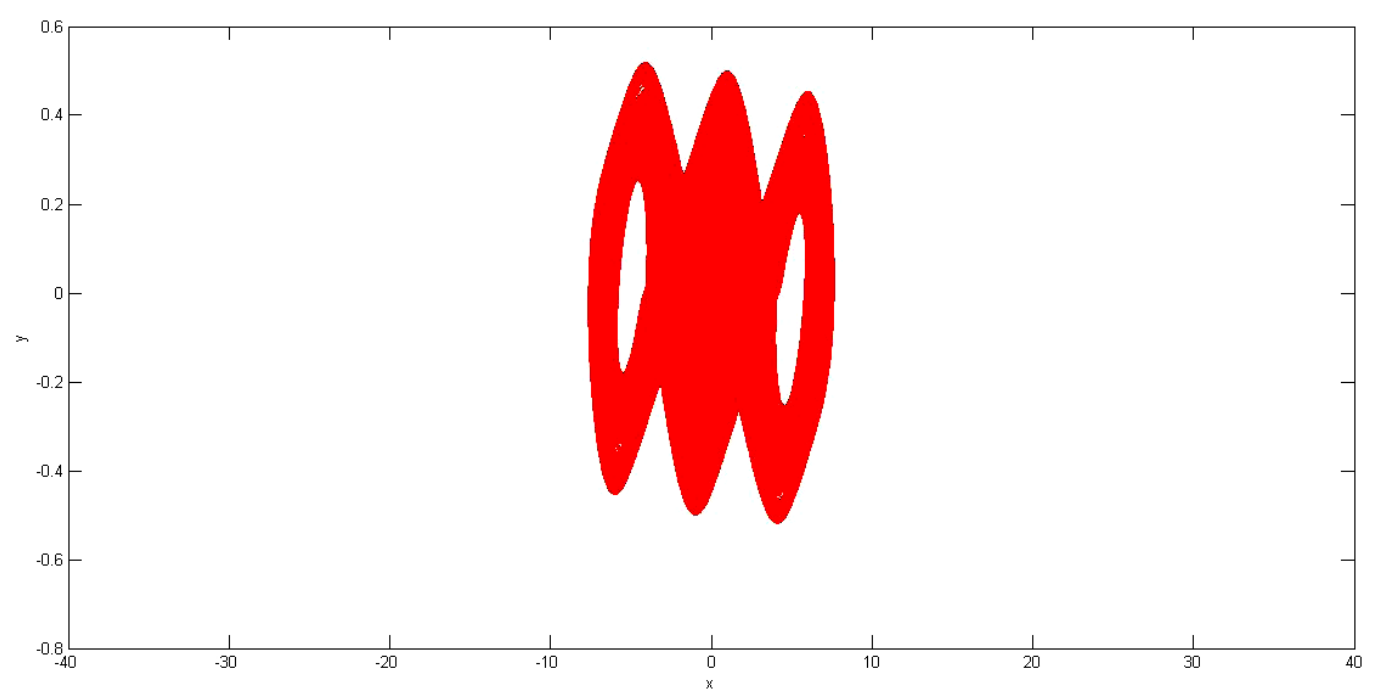

(b)

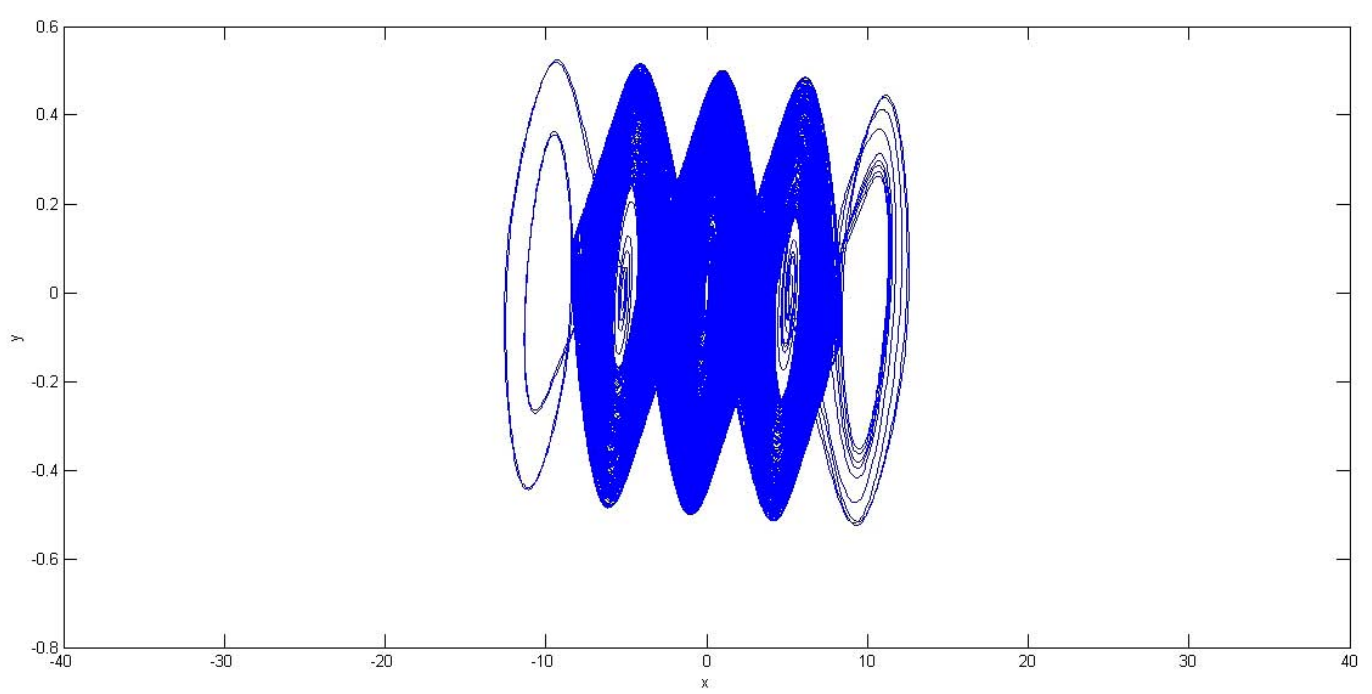

(c)

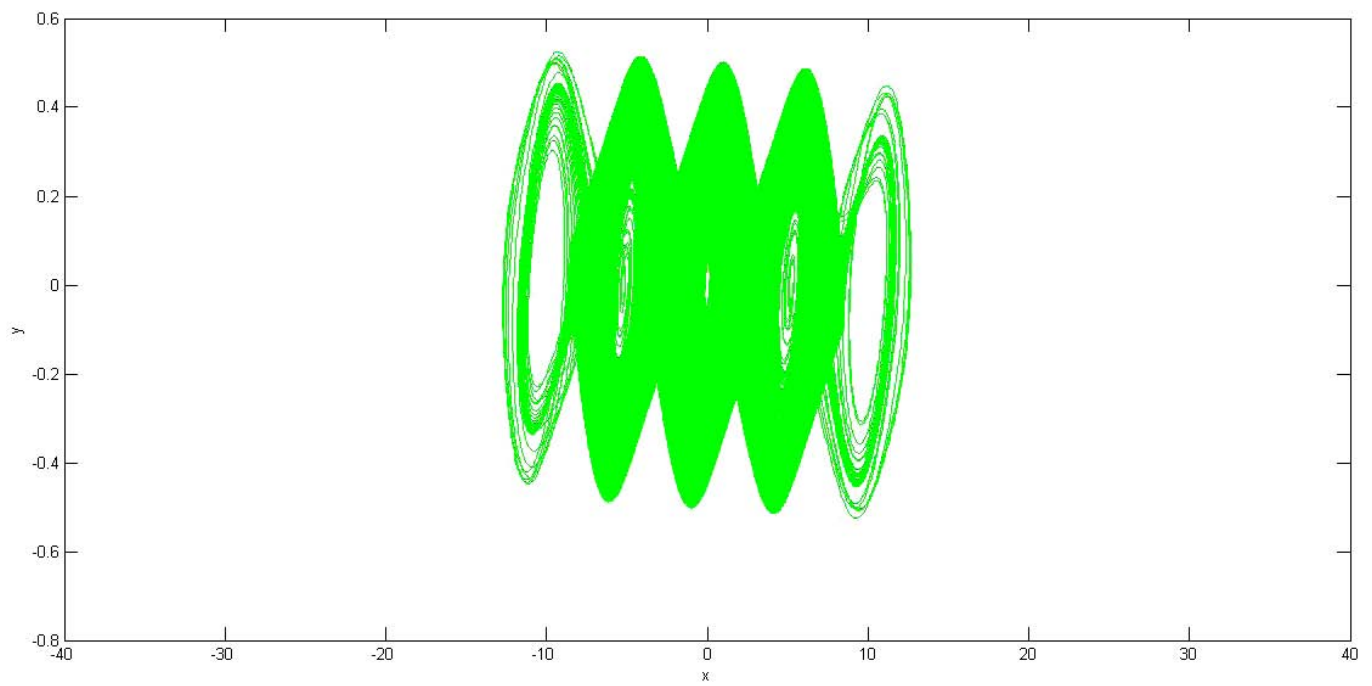

Fig. 8. $n$-scroll (odd scroll) attractors generated by the hidden bifurcation method for $c=4$. (a) 3 spirals for $\varepsilon=0.98$, (b) 5 spirals for $\varepsilon=0.9852$ and (c) 5 spirals for $\varepsilon=0.986$. The range of the $x$-axis is the same for Figs. $5-12$. 
(a)

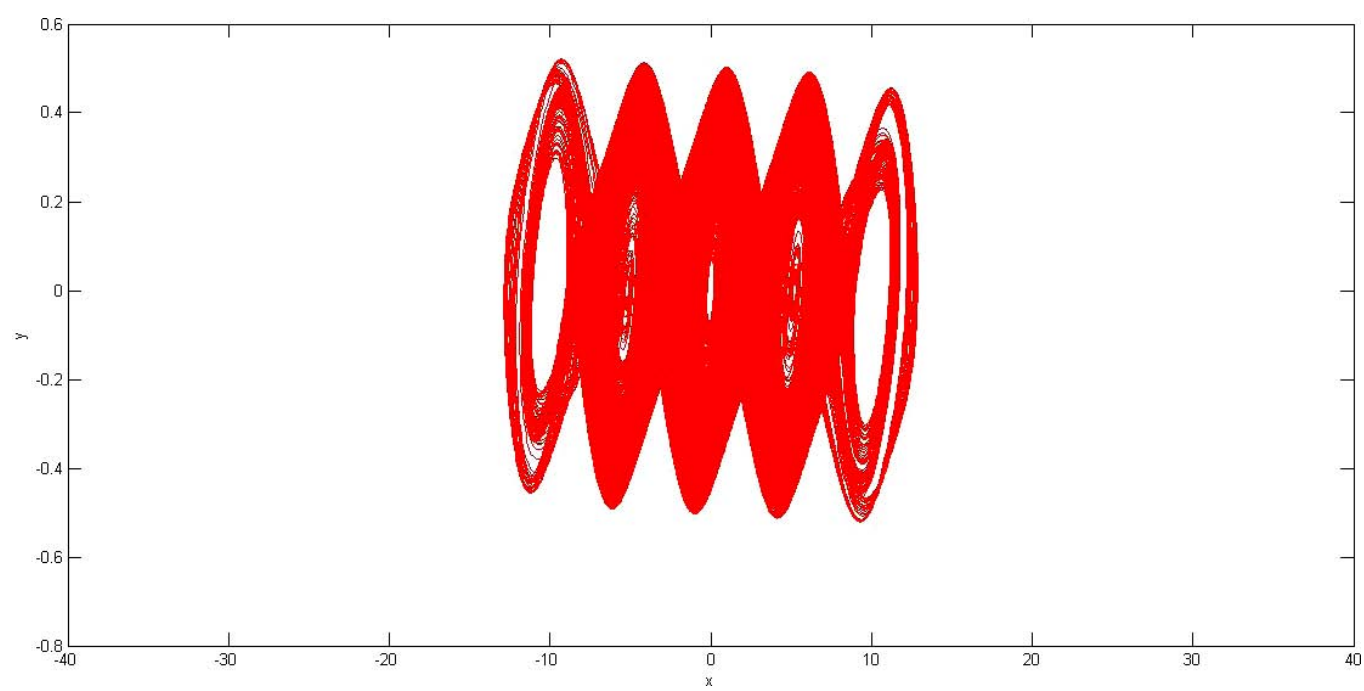

(b)

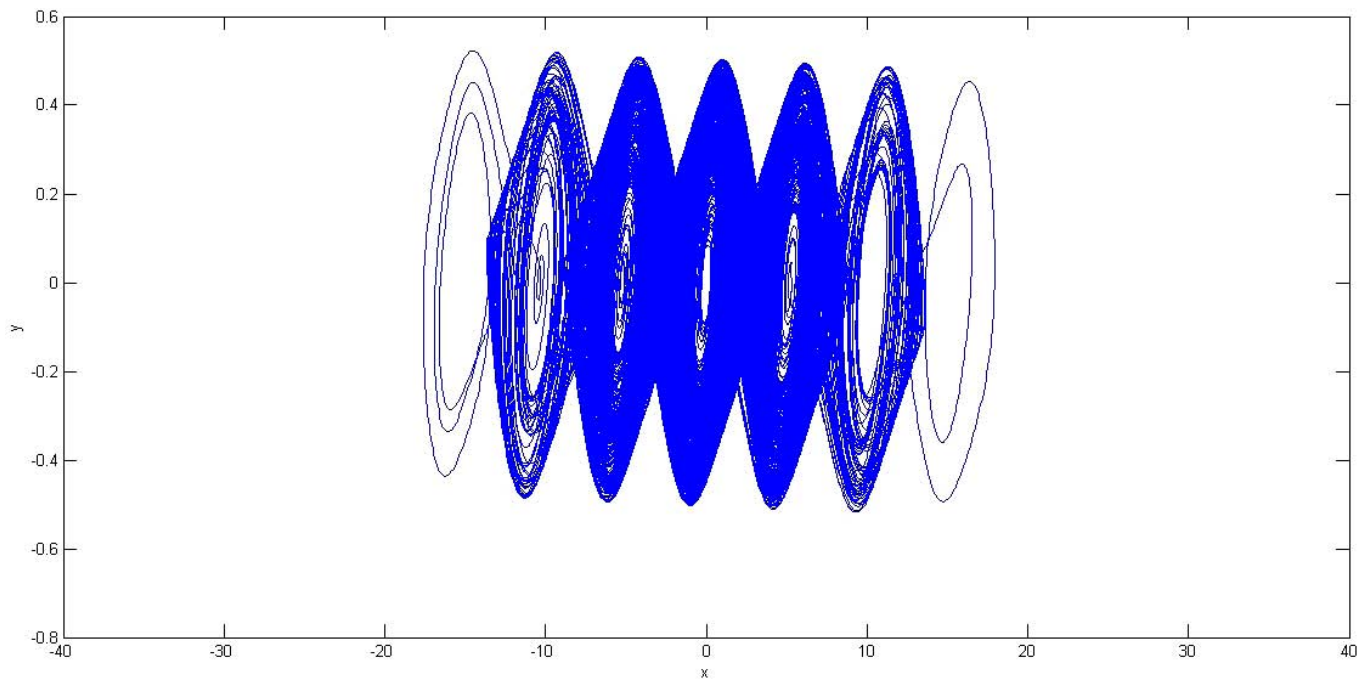

(c)

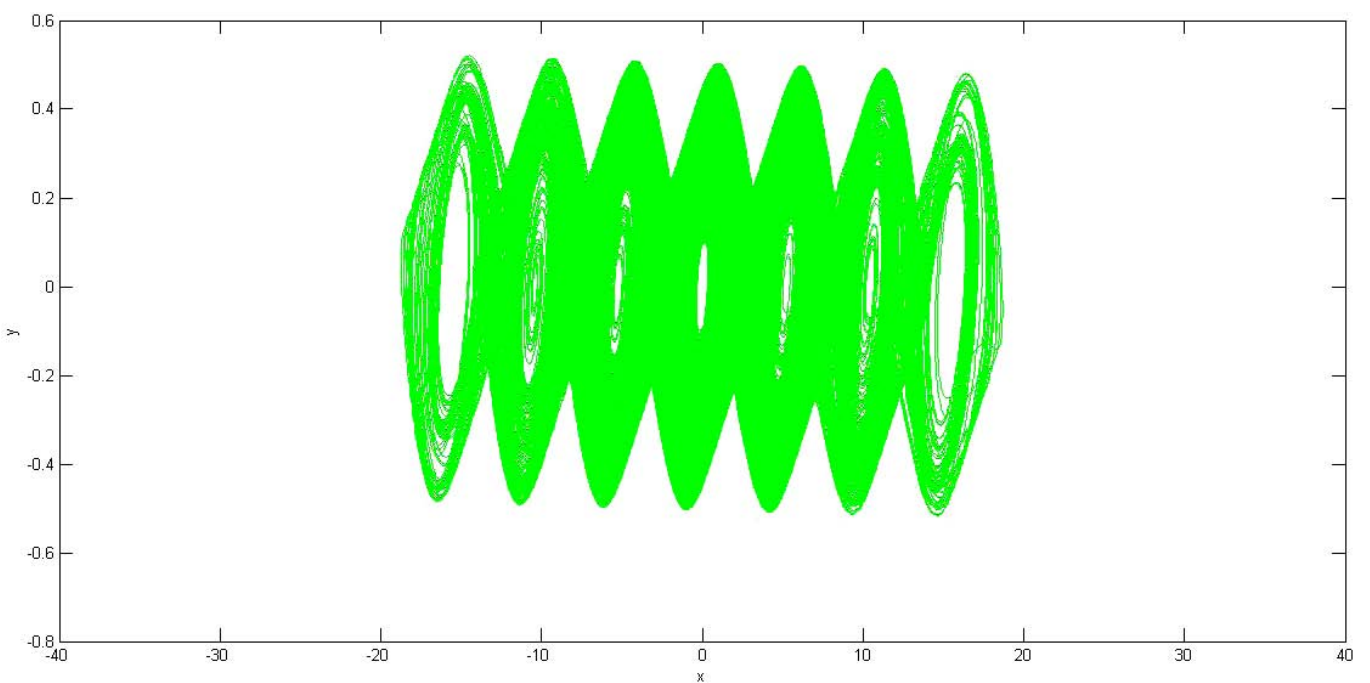

Fig. 9. $n$-scroll (odd scroll) attractors generated by the hidden bifurcation method for $c=6$. (a) 5 spirals for $\varepsilon=0.98$, (b) 7 spirals for $\varepsilon=0.9852$ and (c) 7 spirals for $\varepsilon=0.986$. The range of the $x$-axis is the same for Figs. $5-12$. 
(a)

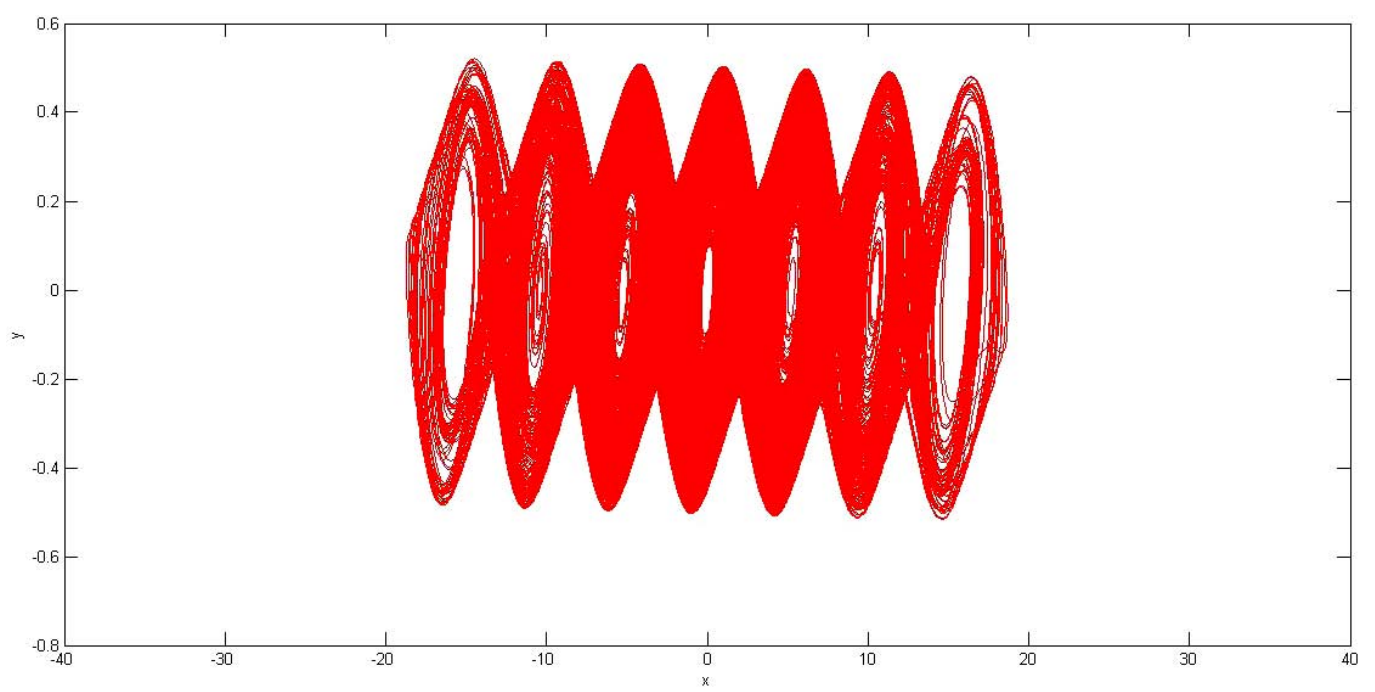

(b)

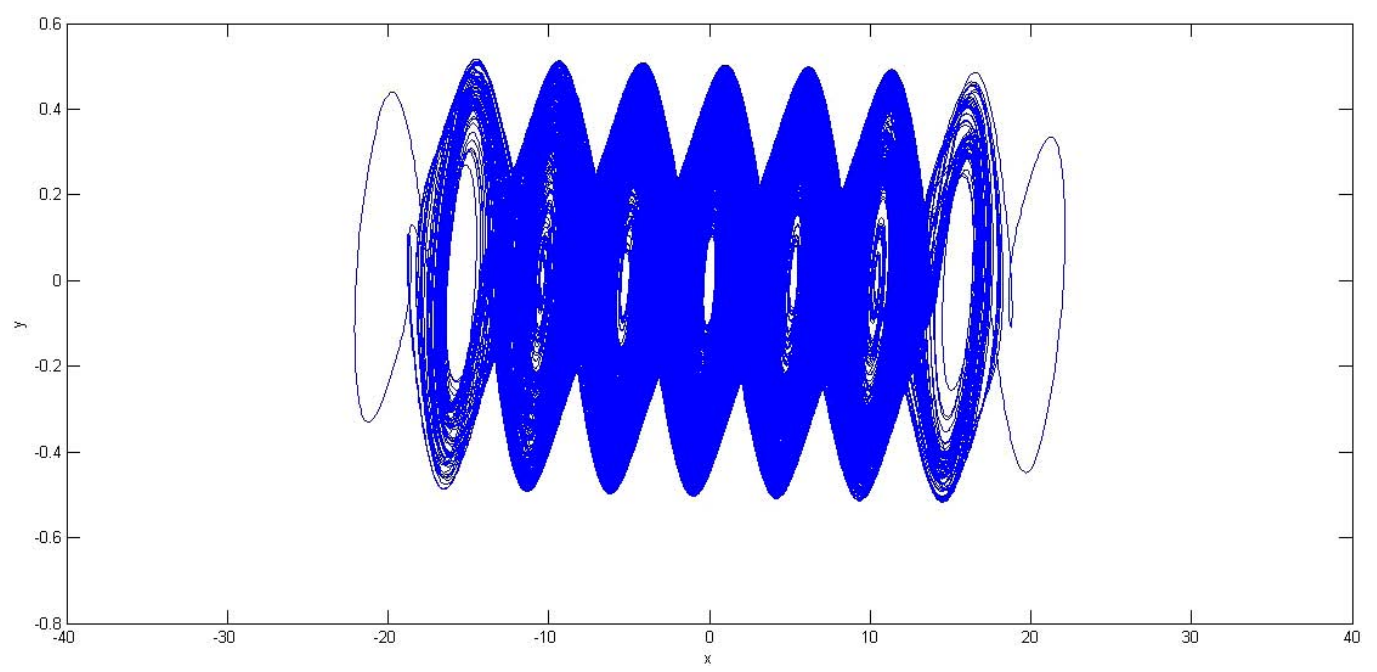

(c)

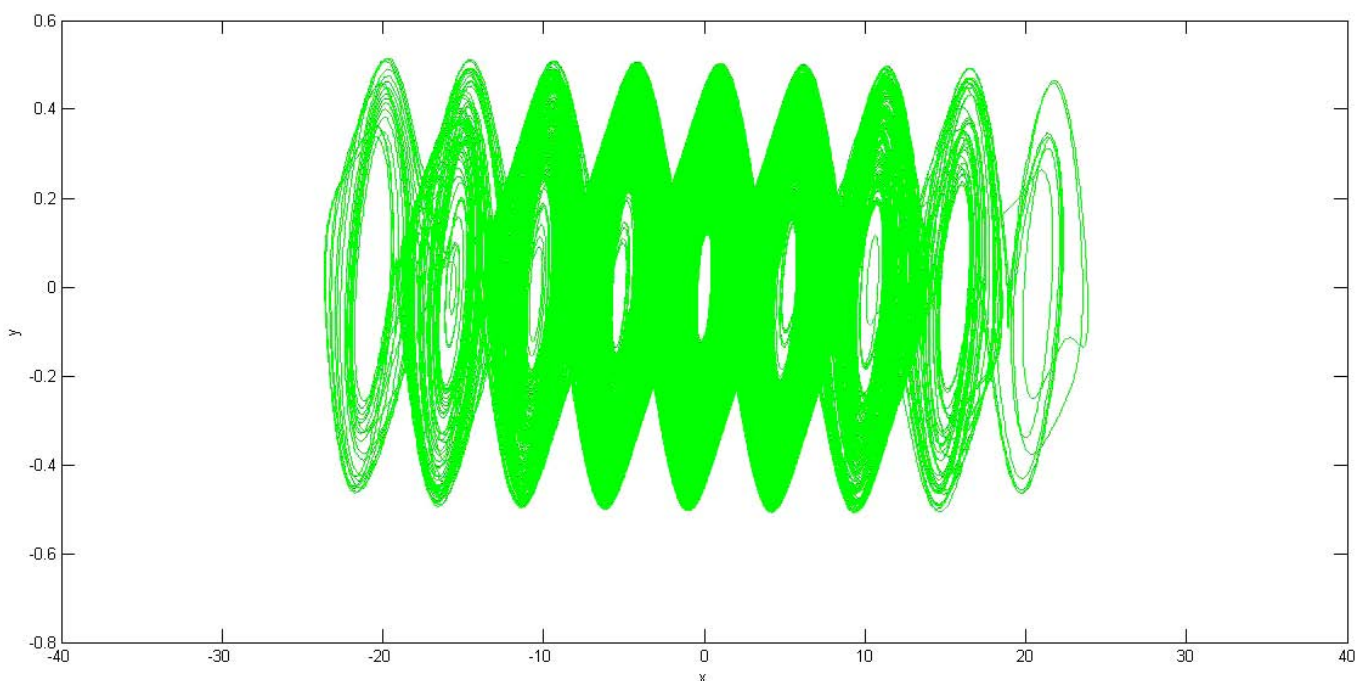

Fig. 10. $n$-scroll (odd scroll) attractors generated by the hidden bifurcation method for $c=8$. (a) 7 spirals for $\varepsilon=0.989$, (b) 9 spirals for $\varepsilon=0.99$ and (c) 9 spirals for $\varepsilon=0.994$. The range of the $x$-axis is the same for Figs. $5-12$. 
(a)

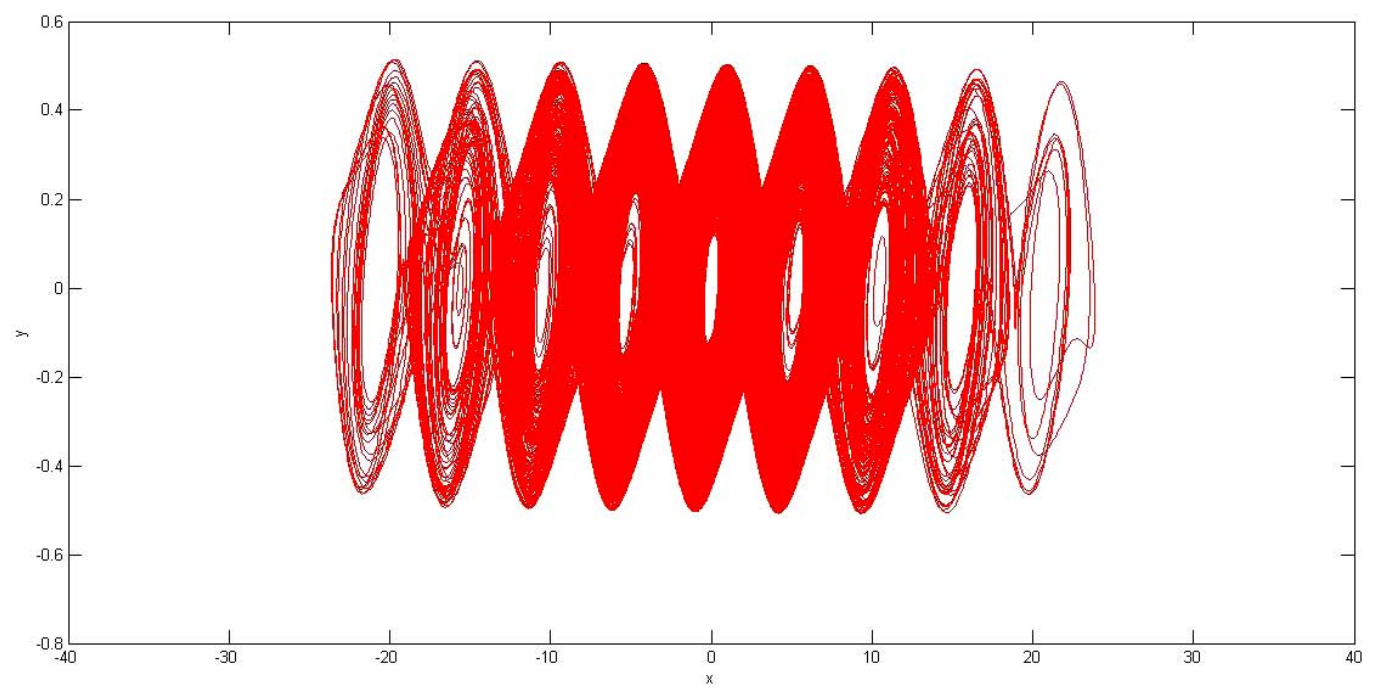

(b)

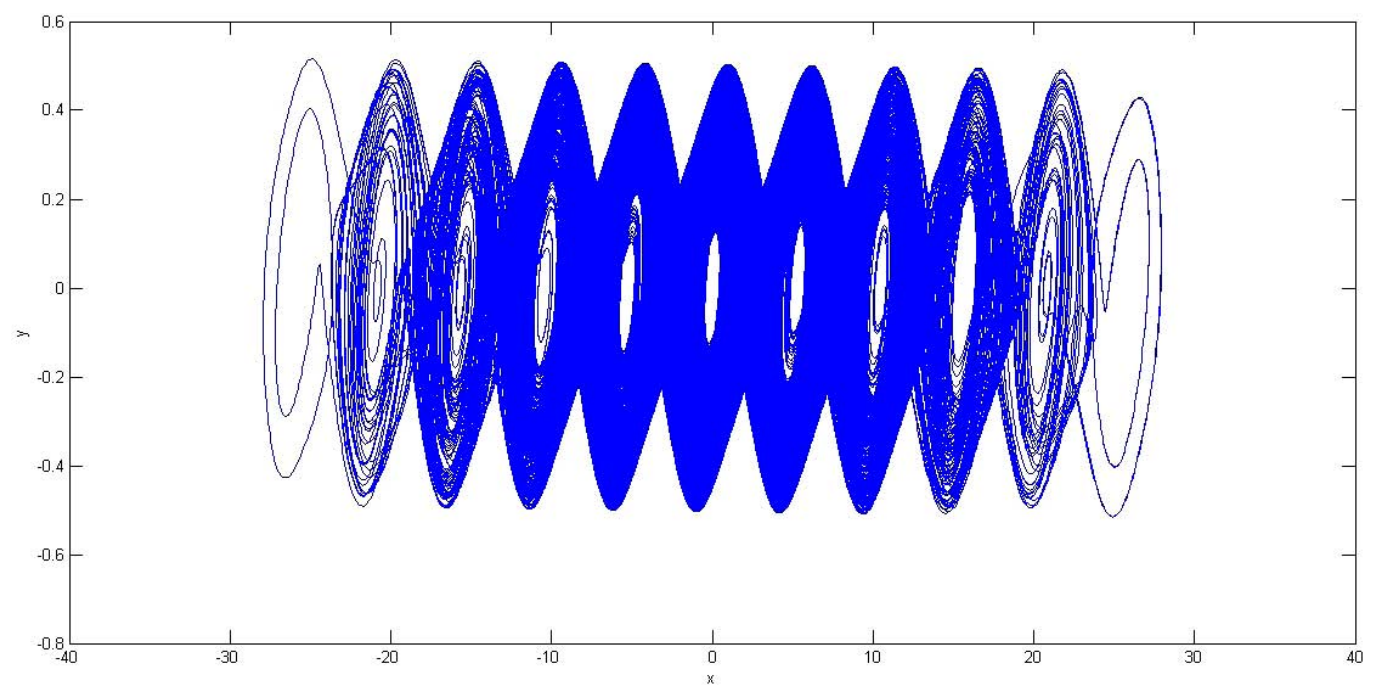

(c)

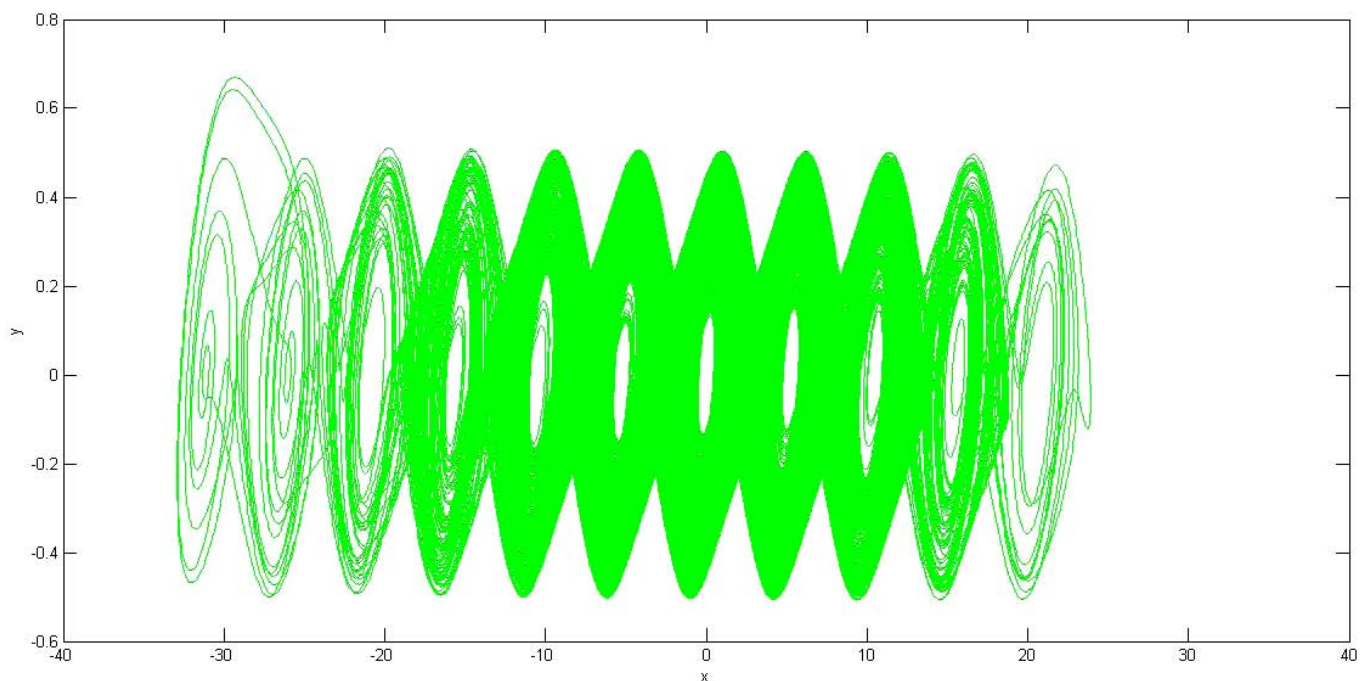

Fig. 11. $n$-scroll (odd scroll) attractors generated by the hidden bifurcation method for $c=10$. (a) 9 spirals for $\varepsilon=0.994$, (b) 11 spirals for $\varepsilon=0.995$ and (c) 11 spirals for $\varepsilon=0.997$. The range of the $x$-axis is the same for Figs. $5-12$. 
(a)

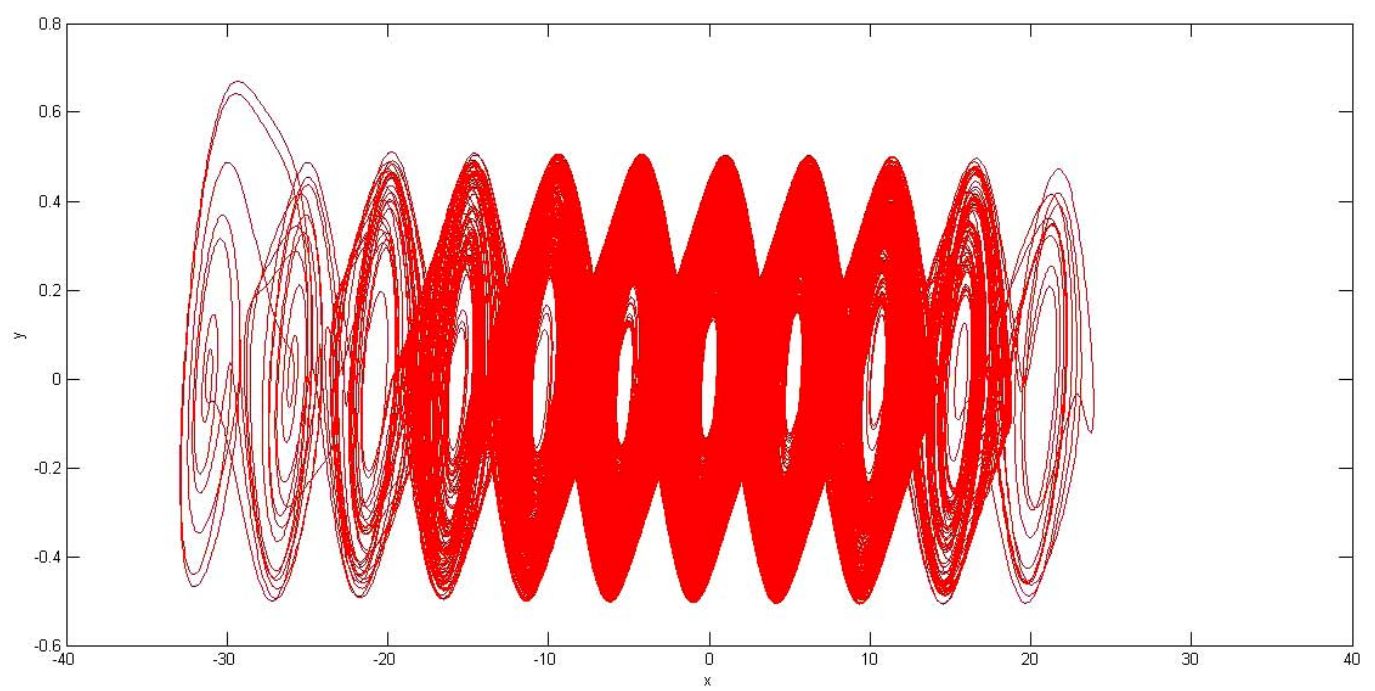

(b)

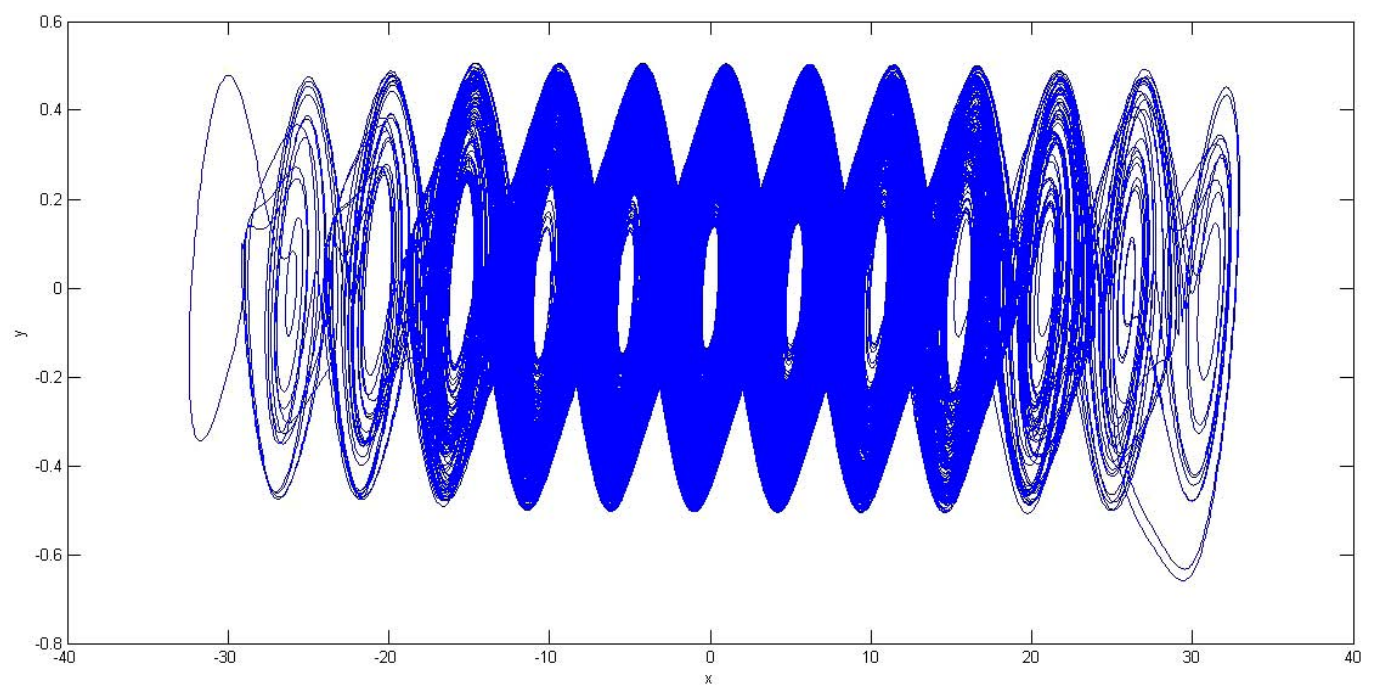

(c)

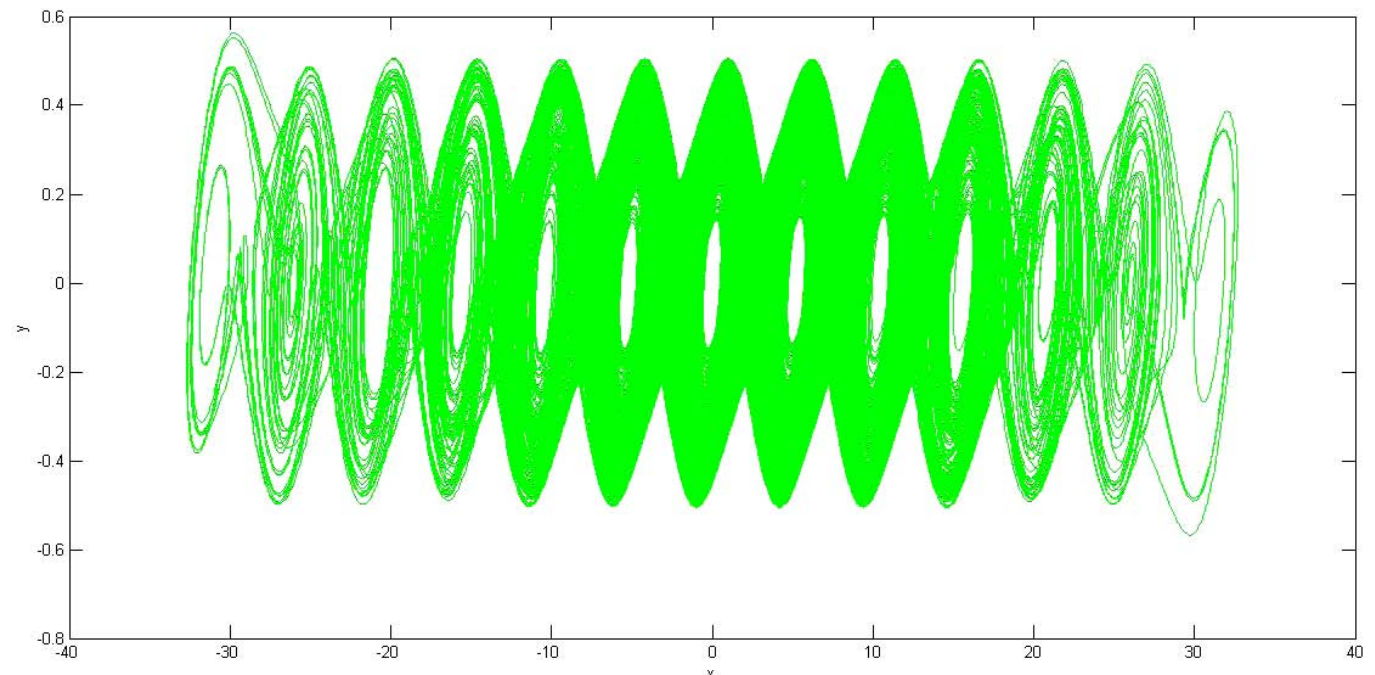

Fig. 12. $n$-scroll (odd scroll) attractors generated by the hidden bifurcation method for $c=12$. (a) 11 spirals for $\varepsilon=0.997$, (b) 13 spirals for $\varepsilon=0.998$ and (c) 13 spirals for $\varepsilon=0.999$. The range of the $x$-axis is the same for Figs. $5-12$. 


$$
\begin{aligned}
& 0 \\
& 0 \\
& 0
\end{aligned}
$$




$$
\begin{aligned}
& 0 \\
& 0 \\
& 0
\end{aligned}
$$


(a)

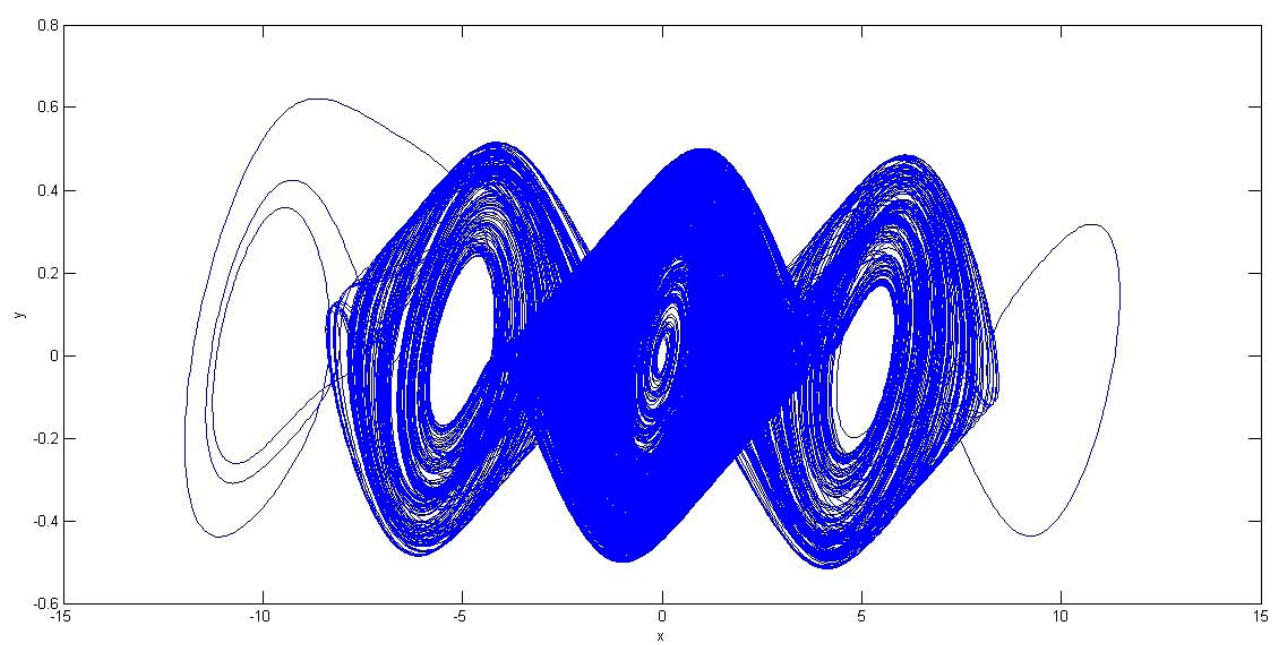

(b)

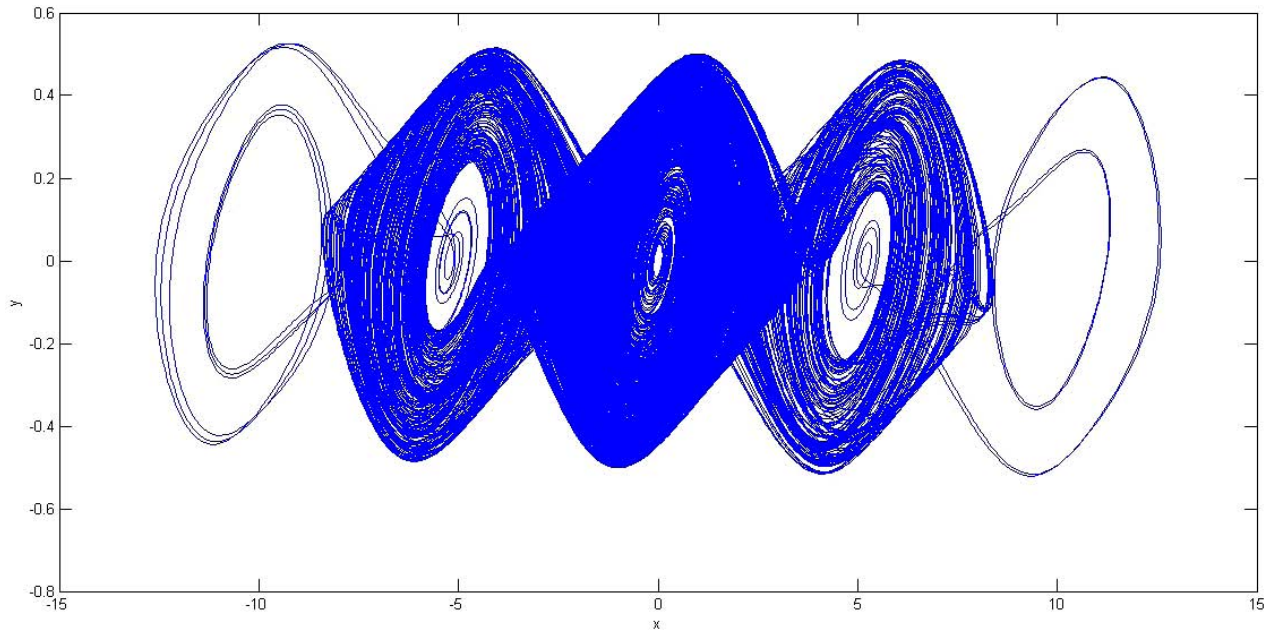

(c)

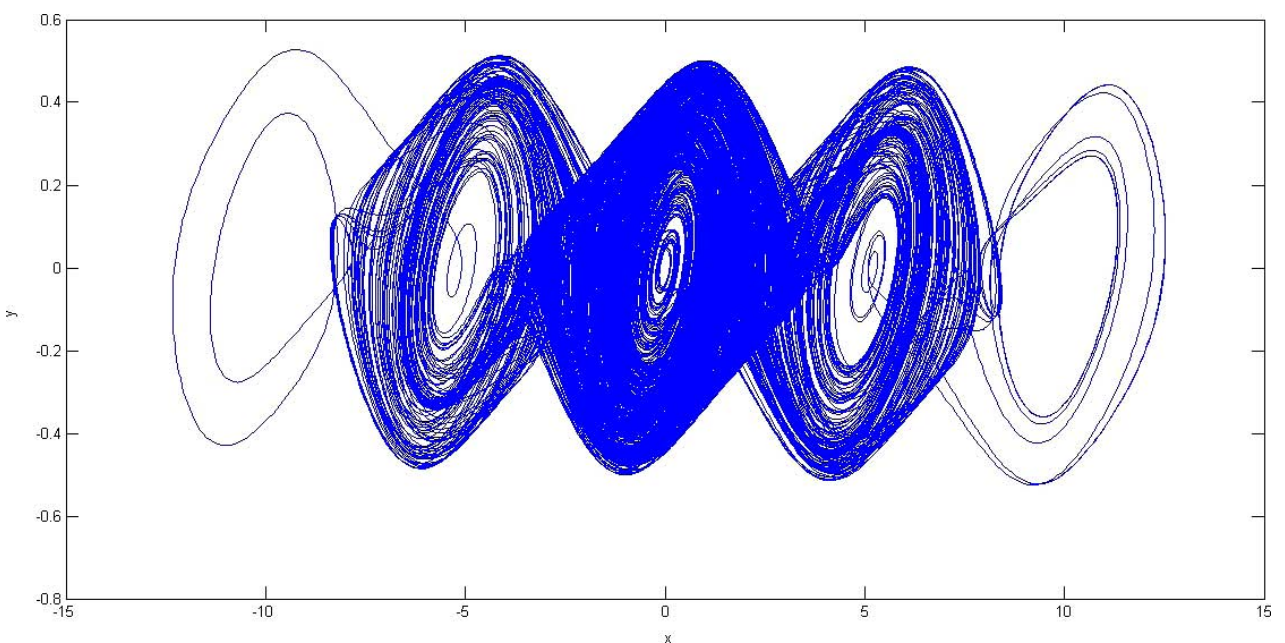

Fig. 14. Comparison between the shape of the attractors for the same value of $\varepsilon=0.9718$, and various values of parameter c. (a) $c=4$, (b) $c=6$, (c) $c=8$, (d) $c=10$ and (e) $c=12$. 
(d)

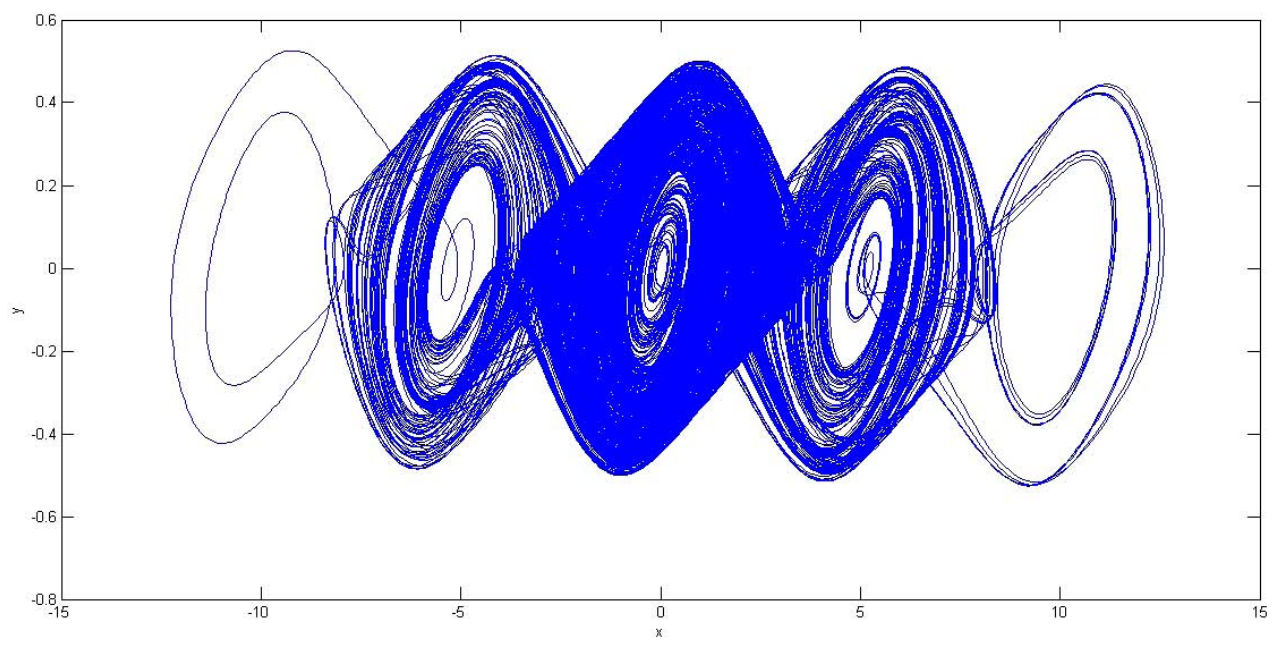

(e)

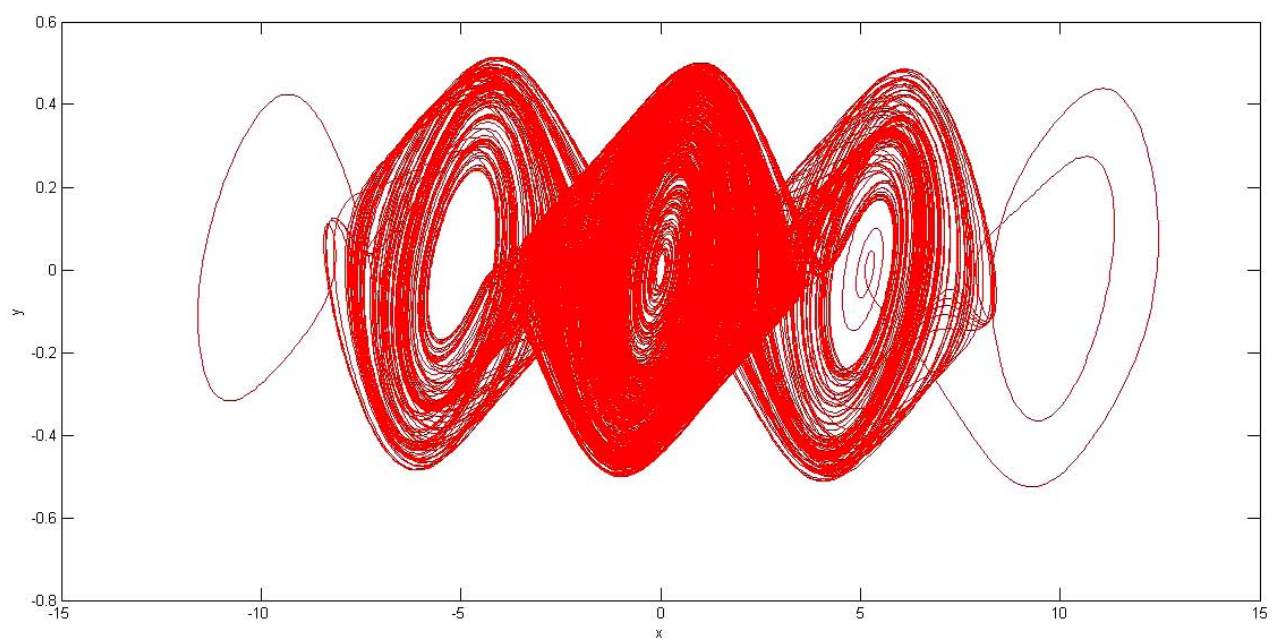

Fig. 14. (Continued)

(a)

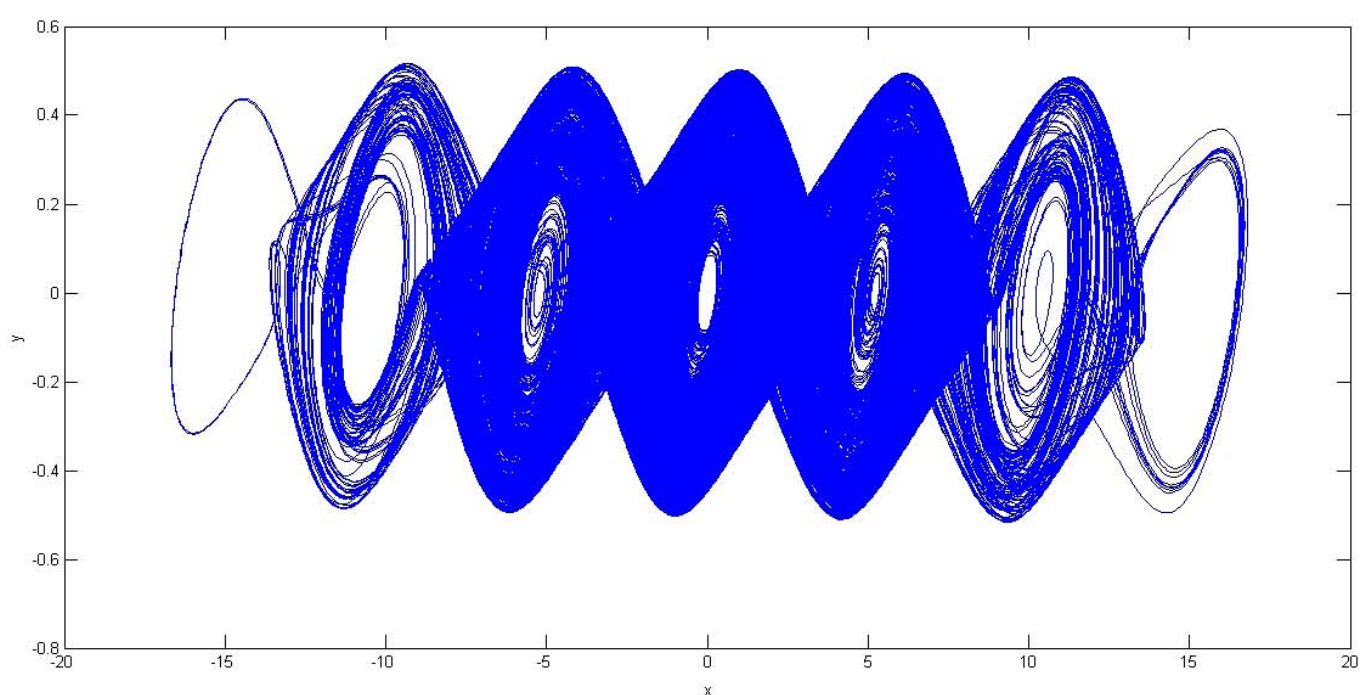

Fig. 15. Comparison between the shape of the attractors for the same value of $\varepsilon=0.9852$, and various values of parameter $c$. (a) $c=6$, (b) $c=8$, (c) $c=10$ and (d) $c=12$. 

(a)

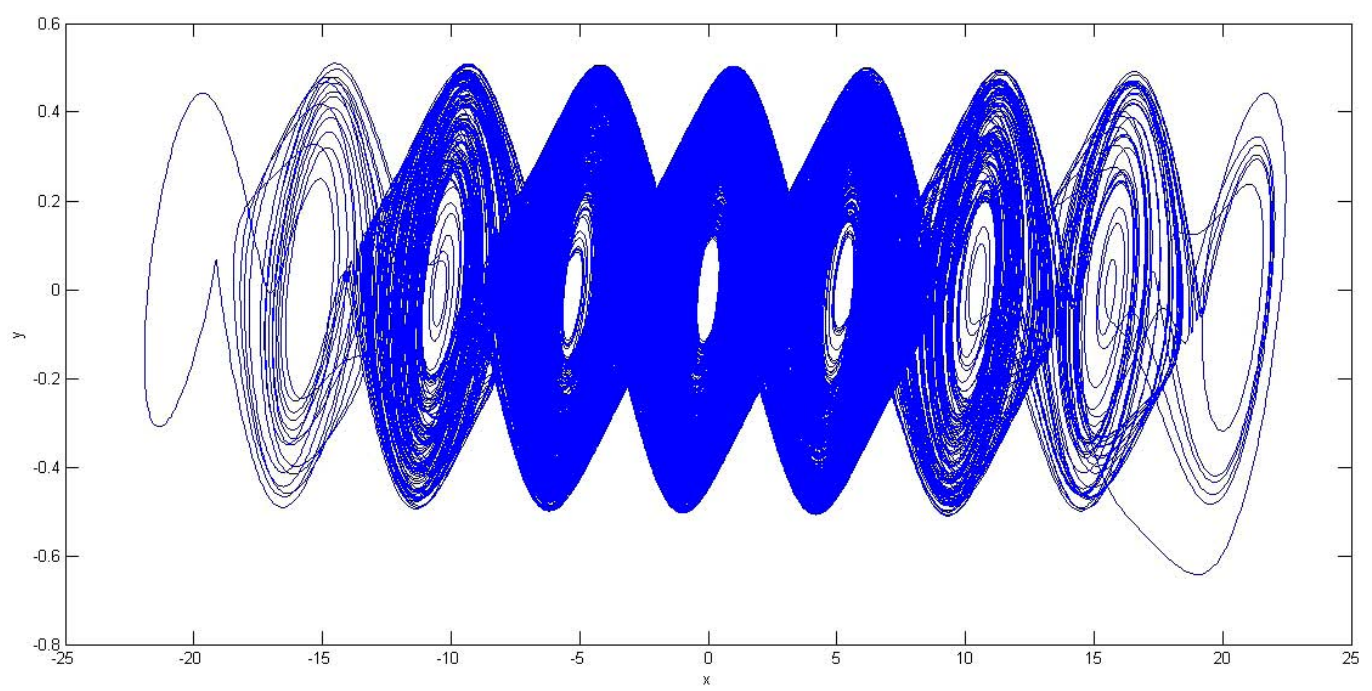

(b)

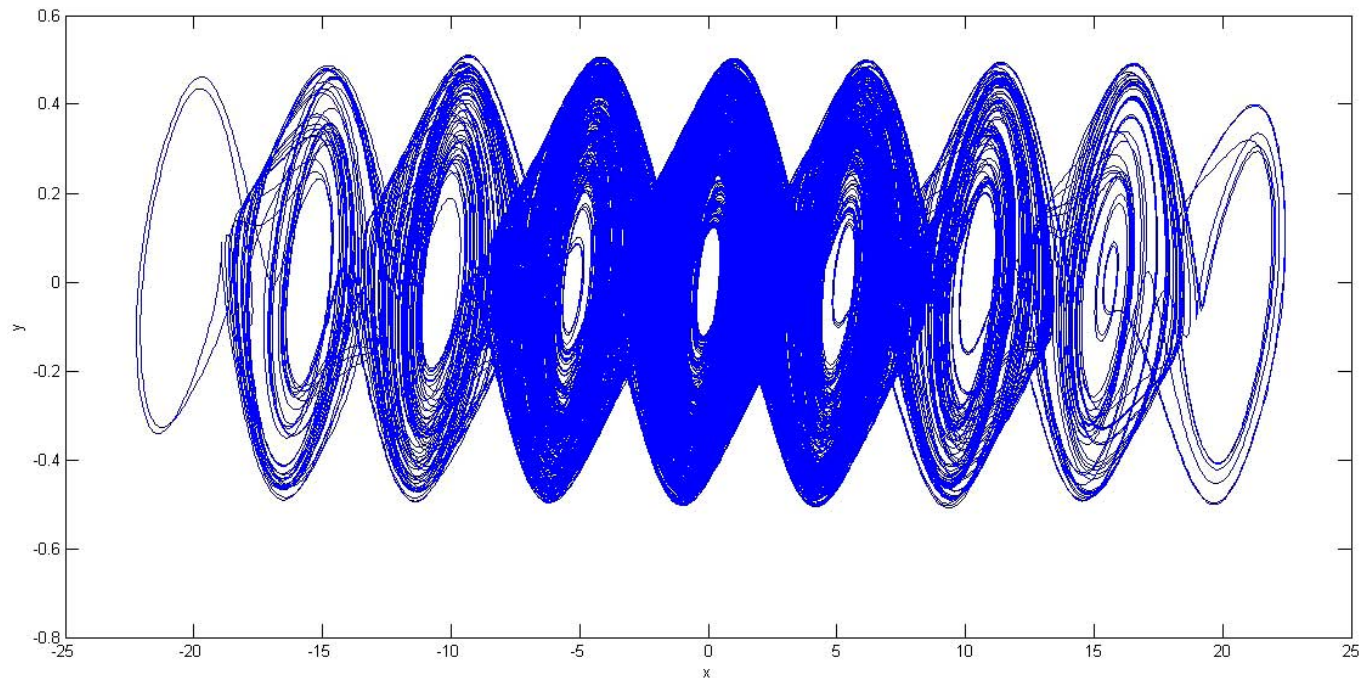

(c)

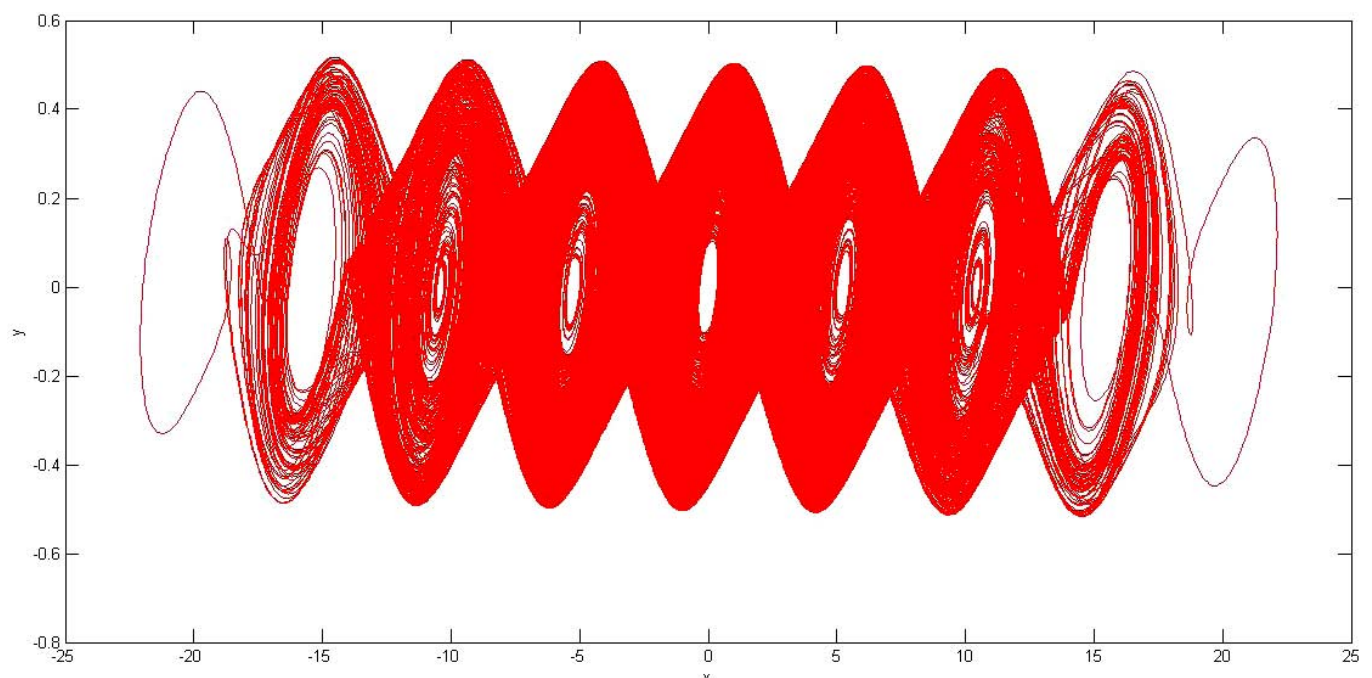

Fig. 16. Comparison between the shape of the attractors for the same value of $\varepsilon=0.99$, and various values of parameter $c$. (a) $c=8$, (b) $c=10$ and (c) $c=12$. 
(a)

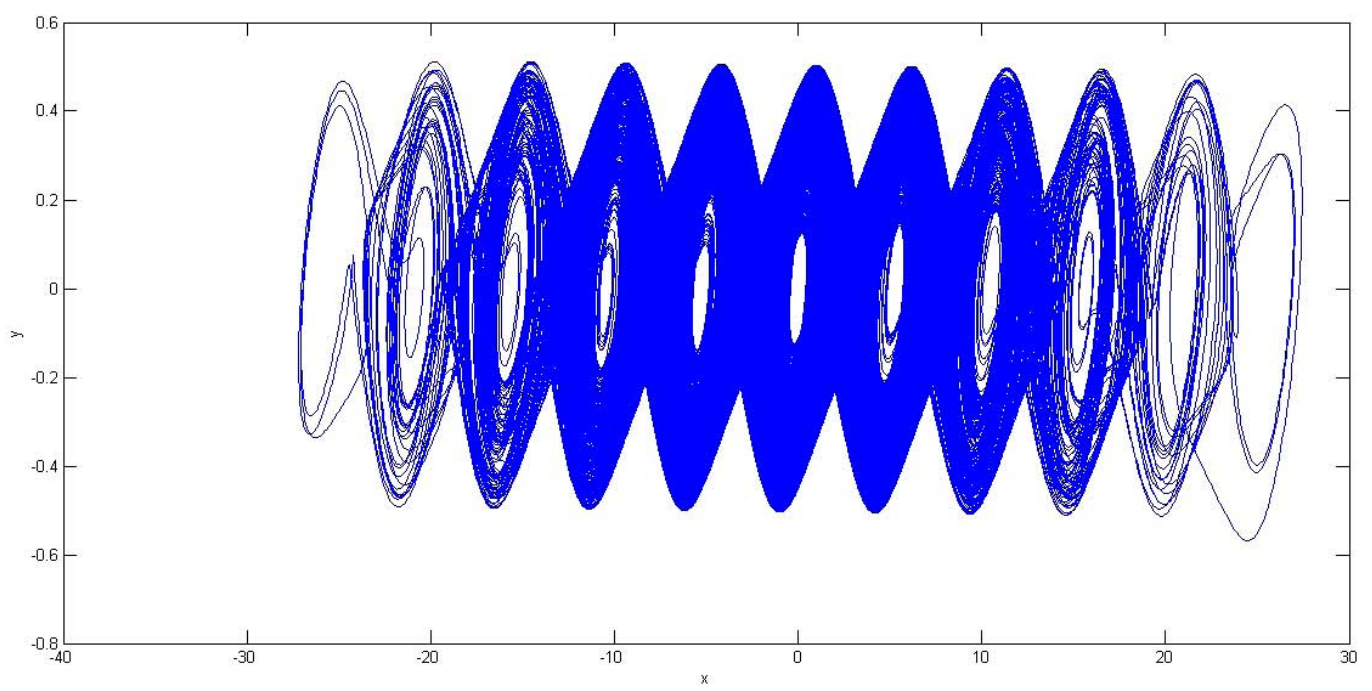

(b)

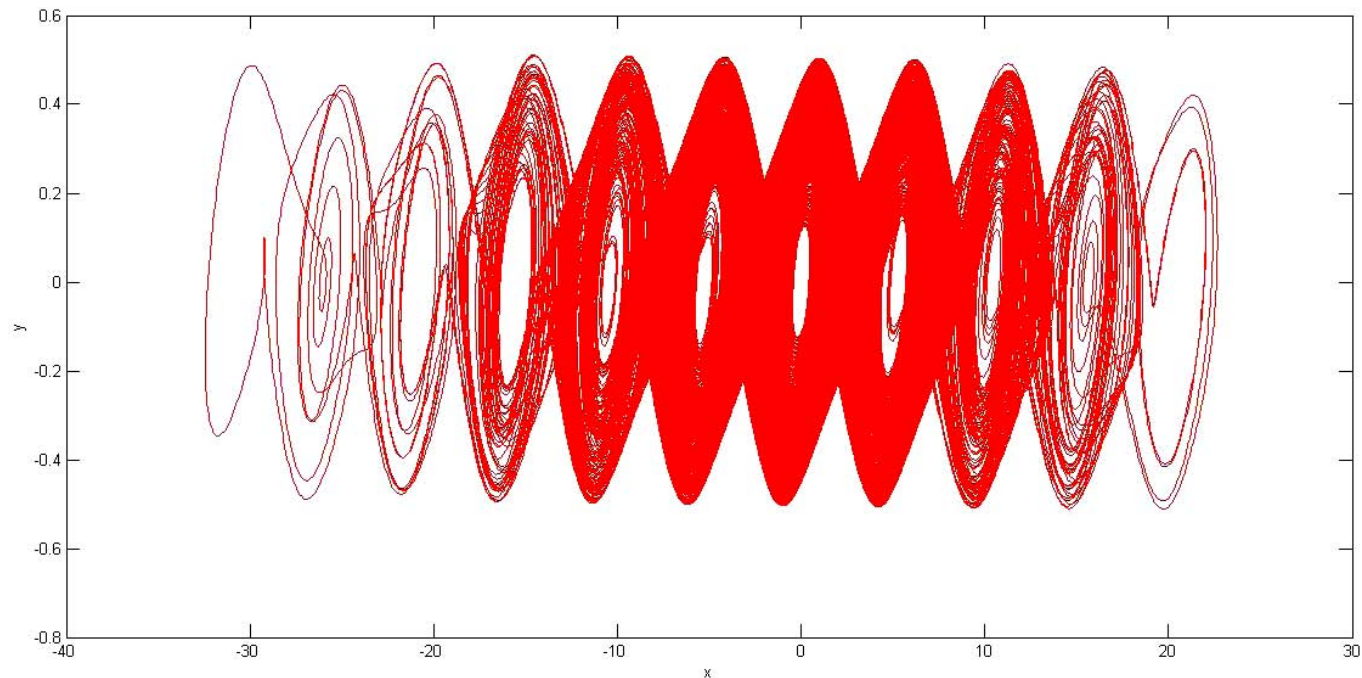

Fig. 17. Comparison between the shape of the attractors for the same value of $\varepsilon=0.995$, and various values of parameter $c$. (a) $c=10$ and (b) $c=12$.

(from $c=1$ to $c=12$ ). These results are obtained integrating the differential equation, using Matlab over a sufficient period of time.

If the integration process is stopped early, only a part of spirals are plotted. The knowledge of this range is obtained numerically by trial-error method.

Note that during those computations no hidden attractor was found.

\subsection{Comparison}

We have made a comparison between the common branch points of the various values of parameter $c$ (i.e. where the same number of spirals was obtained using the same values of the parameter $\varepsilon$ ). Following the numerical simulations and the figures obtained, we note that the attractors are very similar as shown in Figs. 13-17.

\section{Conclusion}

In this paper, we have introduced hidden bifurcations in order to overcome the problem of the use of a discrete parameter instead of a continuous one. Based on the method of Leonov for localization of hidden attractors, we have applied a special analytical-numerical algorithm for hidden bifurcation localization in the Chua multispiral model, by introducing an additional continuous parameter $\varepsilon$ in order to find the hidden bifurcations, points of appearance of new spirals. We have called 
such bifurcations hidden bifurcations because there is a need for an additional parameter to unfold the actual structure of the multispiral attractor. A numerical comparison between common bifurcation points for different values of the parameter $c$ is made. Our method for finding hidden bifurcations is very promising. We will soon apply it to two- and three-dimensional parameters, in the case of Multidirectional Multiscroll Chaotic Attractors [Lü et al., 2006].

\section{Acknowledgment}

This paper is supported in part by AFOSR grant FA 9550-13-1-0136.

\section{References}

Arena, P., Saglio, L., Fortuna, L. \& Manganaro, G. [1996] "Generation of $n$-double scrolls via cellular neural networks," Int. J. Circuit Th. Appl. 24, 241-252.

Chua, L. O., Komuro, M. \& Matsumoto, T. [1986] "The double scroll family," IEEE Trans. Circuits Syst. 33, 1072-1118.

Chua, L. O. [1992] "The genesis of Chua's circuit," Archiv für Elektronik und Ubertragung-stechnik 46, 250-257.

Duan, Z., Wang, J. \& Huang, L. [2004] "Multi-input and multi-output nonlinear systems interconnected Chua's circuits," Int. J. Bifurcation and Chaos 14, 3065-3081.

Hirsch, M. W., Smale, S. \& Devaney, R. L. [2003] Differential Equations, Dynamical Systems and an Introduction to Chaos, 2nd edition (Elsevier, Academic Press, Amsterdam).

Huang, A. S., Pivka, L., Wu, C. W. \& Franz, M. [1996] "Chua's equation with cubic nonlinearity," Int. J. Bifurcation and Chaos 12A, 2175-2222.

Khibnik, A. I., Roose, D. \& Chua, L. O. [1993] "On periodic orbits and homoclinic bifurcations in Chua's circuit with a smooth nonlinearity," Int. J. Bifurcation and Chaos 3, 363-384.

Leonov, G. A. [2009a] "On harmonic linearization method," Dokl. Akad. Nauk. Phys. 424, 462-464.

Leonov, G. A. [2009b] "On harmonic linearization method," Automat. Rem. Contr. 5, 65-75.

Leonov, G. A. [2010] "Effective methods for periodic oscillations search in dynamical systems," Appl. Math. Mech. 74, 37-73.

Leonov, G. A., Vagaitsev, V. I. \& Kuznetsov, N. V. [2010] "Algorithm for localizing Chua attractors based on the harmonic linearization method," Dokl. Math. D 82, 663-666.

Leonov, G. A. \& Kuznetsov, N. V. [2011] "Analyticalnumerical methods for investigation of hidden oscillations in nonlinear control systems," Preprints of the 18th IFAC World Congress, Milano, Italy, August 28 September 2, 2011, pp. 2494-2505.

Leonov, G. A., Kuznetsov, N. V. \& Vagaitsev, V. I. [2011] "Localization of hidden Chua's attractors," Phys. Lett. A 375, 2230-2233.

Leonov, G. A., Kuznetsov, N. V. \& Vagaitsev, V. I. [2012] "Hidden attractor in smooth Chua systems," Physica D 241, 1482-1486.

Lorenz, E. [1963] "Deterministic non periodic flow," J. Atmosph. Sci. 20, 130-141.

Lozi, R. [1975] "A computing method for bifurcation boughs of nonlinear eigenvalue problems," Bull. Amer. Math. Soc. 81, 1127-1129.

Lozi, R. \& Ushiki, S. [1993] "The theory of confinors in Chua's circuit: Accurate analysis of bifurcations and attractors," Int. J. Bifurcation and Chaos 3, 333361.

Lü, J., Yu, S., Lung, H. \& Chen, G. [2006] "Experimental verification of multidirectional multiscroll chaotic attractors," IEEE Trans. Circuits Syst.-I: Reg. Papers 53, 149-165.

Matsumoto, T. [1984] "A chaotic attractor from Chua's circuit," IEEE Trans. Circuits Syst. 31, 1055-1558.

Sattinger, D. H. [1973] Topics in Stability and Bifurcation Theory, Lecture Notes in Math., Vol. 309 (Springer-Verlag, Berlin, Heidelberg).

Shilnikov, L. P. [1994] "Chua's circuit: Rigorous results and future problems," Int. J. Bifurcation and Chaos 4, 489-519.

Suykens, J. A. K. \& Vandewalle, J. [1991] "Quasilinear approach to nonlinear systems and the design of $n$-double scroll $(n=1,2,3,4, \ldots)$, , IEE Proc. G: Circuits Dev. Syst. 138, 595-603.

Suykens, J. A. K., Huang, A. \& Chua, L. O. [1997] "A family of $n$-scroll attractors from a generalized Chua's circuit," Int. J. Electron. Commun. 51, 131138.

Tang, K. S., Zhong, G. Q., Chen, G. \& Man, K. F. [2001] "Generation of $n$-scroll attractors via sine function," IEEE Trans. Circuits Syst. 48, 1369-1372.

Yalçin, M. E., Suykens, J. A. K. \& Vandewalle, J. [1999] "On the realization of $n$-scroll attractors," Proc. 1999 IEEE Int. Symp. Circuits and Systems, Vol. 5, pp. 483-486.

Zhong, G. Q. \& Ayrom, F. [1985] "Experimental confirmation of chaos from Chua's circuit," Int. J. Circuit Th. Appl. 13, 93-98. 